\title{
Delinquenz als soziales Handeln 1
}

\author{
Eine erweiterte Modellierung und empirische Überprüfung
}

\section{Von Rolf Becker}

Zusammenfassung: Im vorliegenden Beitrag wird aus Perspektive der strukturell-individualistischen Handlungstheorie versucht, kriminelles Handeln als soziales Handeln zu modellieren. Die subjektiv rationale Entscheidung für oder gegen delinquentes Handeln erfolgt in Abhängigkeit von Restriktionen, Gelegenheiten, Assoziationen sowie von normativen Überzeugungen und moralischen Bewertungen von Straftaten. Die empirische Anwendung mithilfe einer Bevölkerungsumfrage in Bern liefert für intendierte Straftaten wie Ladendiebstahl, Schwarzfahren, Versicherungsbetrug und Steuerhinterziehung theoriekonsistente Ergebnisse.

\section{Einleitung}

Die Untersuchung von Kriminalität und Delinquenz ist für die Sozialwissenschaften - insbesondere für die Ökonomie und Soziologie - besonders interessant, weil es sich um ein soziales Handeln handelt, das für die kriminellen Akteure zuweilen Gewinne erbringt, aber sowohl für die einzelnen Opfer als auch für die Gesellschaft in der Regel mit negativen Externalitäten einhergeht (Becker 1968). ${ }^{2}$ Obgleich Konsens besteht, dass Kriminalität ein ,normales" soziales Phänomen ist (Durkheim 1976), weil es in allen Gesellschaften vorkommt, ist der Forschungsstand für die Erklärung dieses Phänomens trotz aller Fortschritte in den letzten Jahrzehnten immer noch nicht als zufriedenstellend zu bezeichnen (Becker / Imhof / Raimann 2007). Zwar hat sich der ökonomische Erklärungsversuch, basierend auf Arbeiten von Becker (1968) oder Ehrlich (1973), als zu restriktive Entscheidungs- und Handlungstheorie mit widersprüchlichen Befunden in der empirischen Anwendung erwiesen (Becker / Mehlkop 2006). Aber er wurde in der Zwischenzeit erfolgreich in der Soziologie adaptiert, modifiziert und weiterentwickelt (Wikström 2006; Dahlbäck 2003).

Insbesondere die soziologisch und sozialpsychologisch aufgeklärte Variante, Kriminalität über Delinquenz als subjektiv rationale Wahlhandlung zu erklären, hat sich - über die Verbindung der Theorie subjektiver Werterwartung (Becker / Mehlkop 2006) mit der ,Frame Selection Theory ' (Kroneberg / Heintze / Mehlkop 2010) - inzwischen etabliert (Mehlkop 2011). Des Weiteren haben sich die Bemühungen bewährt, mit diesen beiden Theorien kompatible Erklärungsversuche - wie etwa die Theorie differentieller Assoziation nach Sutherland (1968) oder die Erweiterung der Anomietheorie um Gelegenheitsstrukturen durch Cloward / Ohlin (1961) oder die sozial-kognitive Lerntheorie von Bandura (2001) - in eine Theorie der Delinquenz als soziales Handeln zu integrieren (Becker / Imhof / Raimann 2007).

Anknüpfend an diese jüngere Theorieentwicklung wird im vorliegenden Beitrag eine allgemeine Entscheidungs- und Handlungstheorie des normkonformen Verhaltens bzw. delin-

1 Für wertvolle Hinweise danke ich dem anonymen Gutachter sowie den Herausgebern.

2 Unter Delinquenz wird hier die Neigung verstanden, gesetzesbrechend zu handeln und folglich - wegen Begehens eines strafrechtlich zu verfolgenden Delikts - straffällig zu werden. Mit diesem Begriff wird in der Regel eher auf soziologische Aspekte der Kriminalität (d.h. die Straftaten als Makrophänomen) eingegangen. Juristisch gesehen sind solche Handlungen illegal, weil gegen geltendes Gesetz verstoßen wird. In kriminellen Kreisen hingegen können strafbare Handlungen als legitim (entsprechend den gültigen Gruppennormen als rechtmäßig anerkannt oder moralisch gerechtfertigt) angesehen werden, aber bestimmte Straftaten hingegen von den gleichen Gruppen als illegitim bezeichnet werden, weil etwa gegen eine Konvention oder Norm in dieser Gruppe verstoßen wird. 
quenten Handelns vorgeschlagen und empirisch überprüft. Zunächst werden im zweiten Abschnitt die theoretischen Argumente dargelegt und zu einem allgemeinen Handlungsmodell zusammengefügt. Danach erfolgt die Beschreibung der Datenbasis, der abhängigen und unabhängigen Variablen sowie des angewandten statistischen Verfahrens (dritter Abschnitt). Darauffolgend werden im vierten Abschnitt die empirischen Befunde dargestellt und abschließend im fünften Abschnitt zusammenfassend Schlussfolgerungen daraus gezogen.

\section{Theoretischer Hintergrund}

\section{Gesellschaftliche Verhältnisse}

Wenn zunächst aus Sicht der strukturell-individualistischen Handlungstheorie als Kern einer mechanismenbasierten soziologischen Erklärung davon ausgegangen wird, dass Kriminalität die aggregierte Folge delinquenten Handelns ist, dann ist die Frage zu erklären, wer aus welchen Gründen und unter welchen sozialen Verhältnissen illegale statt legale Handlungen wählt und ausführt (Abb. 1). Gemäß Weber (1922: 12) ist jedes soziale Handeln, folglich auch gesetzesbrechendes Handeln, instrumentell - also ein Ziel verfolgendes und dabei an Verhaltens- oder Handlungsmöglichkeiten anderer Akteure orientiertes Handeln, obgleich es in der Intention, dem „subjektiv gemeinten Sinn“ (Weber 1922: 1) des Handelnden nach, nicht in jedem Fall rein zweckrational sein muss. Wenn das Handeln etwa an Erwartungen anderer Akteure ausgerichtet ist, also an Normen oder Werten orientiert ist, kann es in der Intention als wertrational bezeichnet werden. Wir können generell davon ausgehen, dass Individuen in einer Situation die Handlung wählen, die in ihren Augen mehr Vorteile und weniger Nachteile als andere Alternativen versprechen. Die Vorteile können beispielsweise individuelle Bereicherung (auf Kosten anderer) oder Belohnung infolge einer Entsprechung von Normen und Werten (bei Vermeidung negativer Sanktionen) sein.

Wenn Menschen nach Zielen wie subjektivem Wohlbefinden streben, das sich aus physischer Integrität (Einkommen, Rente) und sozialer Anerkennung (Prestige, Respekt) ergibt, dann werden sie bei gegebenen Gelegenheiten und Restriktionen individueller und struktureller Art (Wikström 2006) über subjektiv angemessenes Handeln versuchen, die Zwischengüter wie Einkommen oder Reputation herzustellen und zu erhalten (Lindenberg 1989; Ormel / Lindenberg / Steverink / Verbrugge 1999). Es sind folglich Individuen, die in solchen Situationen zu strafbaren Handlungen neigen, eine Wahl zwischen legalen und illegalen Tätigkeiten treffen und im Aggregat mit dem Handeln ein bestimmtes Ausmaß der Kriminalität und eine sozialstrukturelle Verteilung von Delinquenz hervorbringen. 
Abbildung 1: Delinquenz und Kriminalität als aggregierte Ergebnisse sozialen Handelns

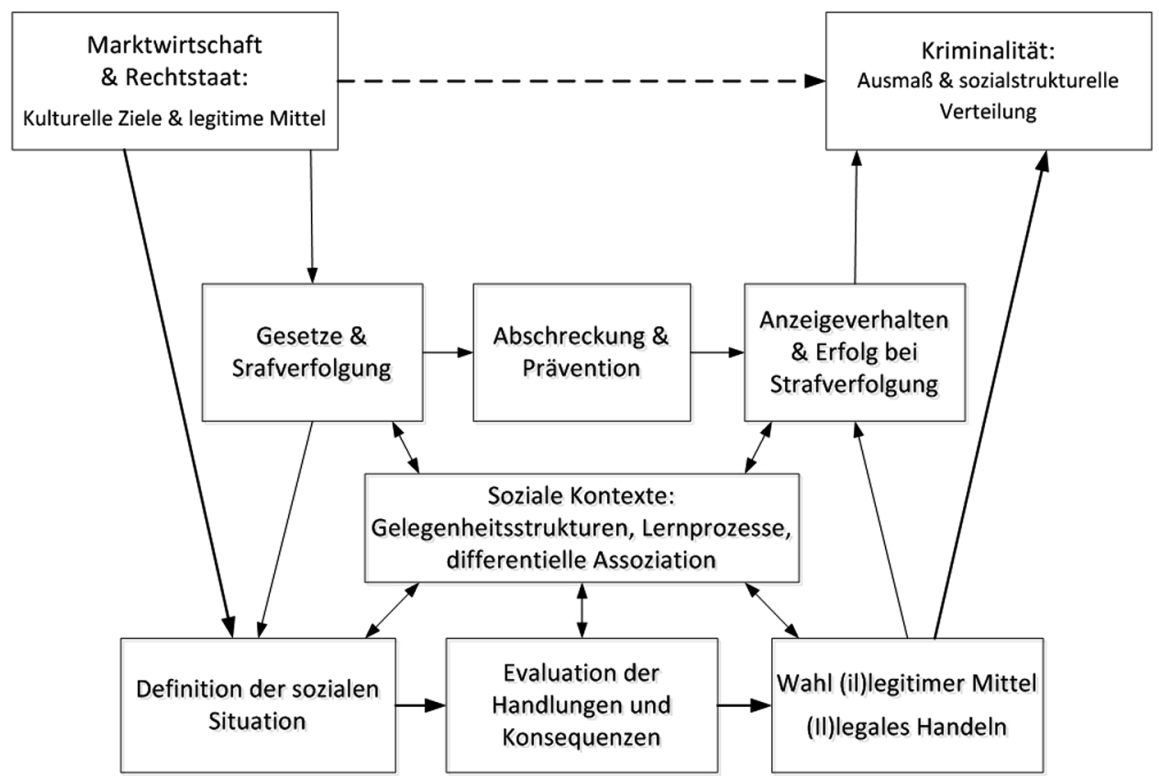

In modernen Gesellschaften existieren zusätzlich zu kulturell definierten Zielen, wie etwa dem Streben nach komfortablem Lebensstandard oder selbständiger Gestaltung des Lebenslaufs, gesellschaftlich definierte Normen, welche die legitimen Mittel und Wege vorgeben, mit denen diese Ziele erreicht werden sollen (Merton 1949). Für die Zielverfolgung gibt es Handlungsweisen (und Mittel), die juristisch als legal oder als strafbar sowie als sozial legitim oder illegitim bezeichnet werden. Die Definition, Regulierung und Kontrolle des normkonformen sowie illegalen Verhaltens obliegt dem Gesetzgeber und der Judikativen. Durch geeignete Maßnahmen und Strategien (z. B. Gesetze, Prävention) versuchen diese korporativen Akteure zum Wohle der Allgemeinheit die Menge illegaler Handlungen in der Bevölkerung (und die negativen Externalitäten) zu minimieren und das Ausmaß legaler Handlungen (bzw. die positiven Externalitäten sozialen Handelns) zu optimieren.

Trotz dieser Rahmenbedingungen beobachten wir, dass Menschen unter bestimmten Gegebenheiten Straftaten begehen - also eine illegale der legalen Handlung vorziehen, weil sie in ihren Augen vorteilhafter oder angemessener erscheint, um ein bestimmtes Ziel wie etwa materielle Bereicherung oder soziale Anerkennung zu erreichen. Da dieses Handeln auf andere individuelle oder korporative Akteure ausgerichtet ist, ist es als soziales Handeln zu verstehen (Weber 1922: 11). Und weil es in der Regel und in den Augen anderer Akteure eher negative Externalitäten in inakzeptablem Maße als positive Folgen für Einzelne und für die Gesellschaft hervorbringt, wird es (vom Gesetzgeber) als illegal definiert (Coleman 1990). Es stellt sich die Frage, warum Menschen dennoch moralische Regeln brechen, obgleich internalisierte Normen und strukturelle Restriktionen in der Weise verhaltensregulierend sein sollten, dass in der Regel normkonformes Handeln zu erwarten wäre (Wikström 2006). Um diese Frage zu klären, wird folgende Theorie vorgeschlagen. 


\section{Das Grundmodell}

Gegeben seien für kognitiv begrenzte, subjektiv rationale Personen die Handlungsalternativen $\mathrm{H}_{\mathrm{i}}$, die jeweils mit einem subjektiv erwarteten Nutzenwert SEU(.) verbunden sind. Der subjektiv erwartete Betrag materiellen Nutzens einer Handlung sei $E$ (z.B. Arbeitseinkommen oder Diebesgut), der subjektiv erwartete Betrag des Verlusts von Reputation sei $-A$ (z.B. Verlust des Status in der eigenen nichtdelinquenten Bezugsgruppe), und der subjektiv erwartete Kostenbetrag sei $-C$ (z.B. Aufwand für Informationsbeschaffung, Planung und Durchführung einer Handlung, Strafmaß als Opportunitätskosten, Beschaffung von Mitteln wie etwa Waffen, Werkzeugen oder Fluchtfahrzeug für die Straftat). ${ }^{3}$ Die subjektive Erwartung eine Handlung erfolgreich auszuführen und den Nutzen E zu realisieren sei als $p$ (d.h. Erfolgswahrscheinlichkeit) definiert und die subjektive Erwartung den Reputationsverlust zu erleiden sei $q$ (etwa die Wahrscheinlichkeit, bei einer strafbaren Handlung entdeckt und sanktioniert zu werden). ${ }^{4}$ Wenn die Handlung, sei sie strafbar oder nicht, erfolgreich sein sollte, dann würde aus Sicht des Akteurs der Nutzen E mit der Wahrscheinlichkeit p eintreten (d.h. p - E). Nur wenn es in den Augen des Akteurs mit der subjektiv erwarteten Wahrscheinlichkeit (1-p) schiefgehen sollte, dann tritt mit einer subjektiv erwarteten Wahrscheinlichkeit q der subjektiv erwartete Reputationsverlust -A (d.h. q ${ }^{-}$A) ein. In diesem Falle wird ein Verlust der Reputation sicher erwartet, der zugleich den materiellen Nutzen einer Handlung verringert und den Kostenbetrag relativ erhöht.

Der subjektiv erwartete Wert des Nichtstuns oder Erduldens $\mathrm{H}\left({ }_{n}\right)$ als Form sozialen Handelns ist: $\operatorname{SEU}\left(\mathrm{H}_{n}\right)=-\mathrm{qA}$. Der subjektiv erwartete Wert einer legalen oder strafbaren Handlung kann formalisiert werden: $\operatorname{SEU}\left(\mathrm{H}_{\mathrm{a}}\right)=\mathrm{pE}+(1-\mathrm{p})(-\mathrm{qA})-\mathrm{C}$. Es wird von einer Person die Handlung $\mathrm{H}_{\mathrm{i}}$ am wahrscheinlichsten ausgeführt, die ihr unter den für sie in Betracht kommenden Handlungsalternativen $\mathrm{H}_{\mathrm{i}}$ den höchsten $\operatorname{SEU}\left(\mathrm{H}_{\mathrm{i}}\right)$-Wert verspricht. ${ }^{5}$ So wird eine legale oder illegale Handlung $\mathrm{H}_{\mathrm{a}}$ dem alternativen Handeln $\mathrm{H}_{n}$ vorgezogen, wenn gilt: $\mathrm{H}_{\mathrm{a}}>\mathrm{H}_{\mathrm{n}}$ bzw. $\mathrm{pE}+(1-\mathrm{p})(-\mathrm{qA})-\mathrm{C}>-\mathrm{qA}$.

Eine mathematische Transformation erleichtert die Interpretation des subjektiven Wertes einer Handlungskonsequenz (Esser 1999: 270): E+qA > C/p. Den Term $E+q A$ nennen wir Handlungsmotivation, was bei strafbaren Handlungen als „kriminelle Energie“ angesehen wird, und C/p als Handlungsrisiko. Die Handlungsmotivation ist umso größer, je höher der materielle Nutzen sowie je höher und wahrscheinlicher die soziale Anerkennung als Handlungskonsequenz ist. Eine Handlung, bei der eine negative Sanktionierung hoch und sicher ist, senkt die Handlungsmotivation. Für eine strafbare Handlung kann die Handlungsmotivation hoch sein, wenn es gilt, als Mitglied einer Autoschieberbande ein wertvolles Auto zu stehlen, was von den Bandenmitgliedern eher mit Anerkennung belohnt wird als die Repara-

3 Die Kosten werden nicht in jedem Fall erwartet - etwa wenn sie in den Augen des Akteurs vernachlässigbar klein erscheinen. Wer bereits aus Erfahrung über die nötigen Informationen für eine Handlung oder Routinen für die Entscheidung oder bereits über die nötigen Mittel für die Handlung verfügt, für den erscheinen die Kosten einer Handlung niedrig. Bei Straftaten werden oftmals Strafhöhen als Opportunitätskosten wegen Unkenntnis entweder unterschätzt oder erst gar nicht kalkuliert (Pogarsky et al. 2004).

4 Abweichend vom Vorschlag von Becker (1968; Block / Lind 1975: 241), wird im Falle einer strafbaren Handlung die Entdeckungswahrscheinlichkeit nicht als Komplement der Erfolgswahrscheinlichkeit definiert (vgl. Becker / Mehlkop 2006). Denn sie hängt nicht nur vom eigenen Geschick der normverletzenden Person ab, sondern auch vom Verhalten der Strafverfolgung und dem Anzeigeverhalten von Opfern oder Zeugen.

5 Im Unterschied zur ökonomischen Theorie der Kriminalität wird nicht davon ausgegangen, dass Straftäter den Nutzen maximieren wollen, sondern dass sie in den Grenzen ihrer Fähigkeiten, Gelegenheiten, Ressourcen und moralischen Verpflichtungen die Handlung am ehesten wählen, die ein für sie zufriedenstellendes Optimum erbringt (Ward / Stafford / Gray 2006: 572). 
tur eines Fahrzeugs als ehrbarer Mechaniker in einer Hinterhofgarage. ${ }^{6}$ Wenn der Autodiebstahl schiefgeht, dann ist die negative Sanktionierung (Spott der „Kollegen“, Missbilligung durch Familie und Strafmaß der Rechtsprechung) gewiss und die Höhe hängt u. a. vom „Image“ der Straftat oder von der dafür vorgesehenen Strafe ab. ${ }^{7}$

Die Gewissheit dafür, die Wahrscheinlichkeit q, hängt von der subjektiv erwarteten Entund Aufdeckung der Straftat ab. Die antizipierte Höhe wird durch die erwartete Sanktionierung durch die eigene Bezugsgruppe $(-\mathrm{A})$ und/oder durch die Judikative $(-\mathrm{C})$ bestimmt. Das Handlungsrisiko kann hoch sein, wenn eine Bankfiliale überfallen werden soll und das Individuum über keine Kompetenzen und Mittel dafür verfügt. Hingegen ist es für einen Bankangestellten niedrig, wenn ein Anteil des Zinseszinses von Bankkonten der Kunden auf das eigene Konto transferiert wird. Allerdings kann eine delinquente Person die Erfolgswahrscheinlichkeit erhöhen und zugleich die Entdeckungswahrscheinlichkeit minimieren, indem sie eine Zielgruppe auswählt, die als „leichtes“ Opfer gilt. Allerdings können sich typische Zielpersonen, die infrage kommenden Opfer, selbst durch Prävention vor Straftäter schützen oder sich mit offensivem Anzeigeverhalten gegen Straftäter wehren, so dass für Täter die Erfolgswahrscheinlichkeit und Gewinne gering, aber die Entdeckungswahrscheinlichkeit und Verluste entsprechend hoch sind (Cook 1986).

Eine legale Handlung bedeutet jedoch keinen Ausschluss einer illegalen Handlung, sondern diese Handlungen können sich ergänzen (Lochner 2004: 818). Denn ohne ein zu versteuerndes Einkommen, das durch legale Arbeit erworben wurde, kann eine Person auch keine Steuerhinterziehung begehen. Es gibt auch Äquivalente bei legalen und illegalen Tätigkeiten. So wie bei der Erwerbstätigkeit gibt es Straftaten, die keine besonderen Qualifikationen voraussetzen, während es wiederum kriminelle Tätigkeiten gibt, die mit hohen Qualifikationsanforderungen verbunden sind. Es wird bei gegebenen subjektiv erwarteten Kosten davon ausgegangen, dass der Nutzen sich aus dem materiellen Vorteil des erworbenen Guts (pE) selbst ergibt und - wenn alles gut geht - aus der sozialen Anerkennung (qA) (positive Sanktionierung). Wenn es schiefgeht, und das kann sowohl bei legalen als auch bei kriminellen Handlungen der Fall sein, dann tendiert nicht nur der materielle Nutzen, sondern auch die soziale Anerkennung mit hoher Wahrscheinlichkeit q gegen 0 und - in den Augen des handelnden Akteurs - ist eine negative Sanktionierung -qA (d.h. Verlust von Reputation) wahrscheinlich (Matsueda 2013). Richtung und Grad der sozialen Anerkennung können auch nach sozialer Assoziation differieren und dann in Abhängigkeit von einer normkonformen oder delinquenten Bezugsgruppe als positive oder negative Sanktionierung auftreten (Zhang / Messner 2000). So mag beispielsweise der materielle Nutzen eines Diebesgutes gering sein, aber die Anerkennung der Straftat an sich hoch sein. Umgekehrt kann bei einer strafbaren Handlung der materielle Wert hoch erscheinen, aber der Erhalt der Reputation wahrscheinlich gering sein. Kurzum: die positive bzw. negative Sanktionierung (d.h. der Erhalt bzw. der Verlust von Respekt und Ansehen sowie Scham und Selbstvorwürfe als weite-

6 Der psychische Nutzen für einen Akteur, der mit Begriffen wie, sex and excitement ' oder, sneaky thrills ' umschrieben wird, ist in der Regel kein Selbstwert, sondern wird erst durch dritte Personen geschaffen, die von dieser Straftat erfahren und diese bewerten.

7 Gleiches gilt übrigens auch für legales Handeln, das schiefgehen kann. Wenn zum Beispiel Anstrengungen, in Orientierung an der eigenen Bezugsgruppe ein prestigeträchtiges Bildungszertifikat zu erwerben, aufgrund unzureichender Leistungen nicht zum erwarteten Erfolg führen, ist für die Kinder und ihre Eltern eine beschädigte Reputation (z.B. Schadenfreude anderer Personen in der gleichen Statusgruppe - etwa akademische Kreise) oftmals gewiss (Stocké 2010). Oder häufige Arbeitslosigkeit kann ebenfalls zum Verlust der Reputation als Arbeitskraft führen, wenn sie nicht auf strukturelle Ursachen, sondern auf individuelle Unzulänglichkeiten einer Person zurückgeführt wird (LudwigMayerhofer 2005). 
re emotionale Konsequenz daraus) wird mit -qA als eine Handlungskonsequenz systematisch berücksichtigt (Matsueda / Gartner / Piliavin / Polakowski 1992: 767).

\section{Subjektiver Sinn und ,frame selection“}

Die Entscheidung für oder gegen eine illegale Handlung ist zielorientiert, am Erfolg der intendierten Handlung ausgerichtet. Folglich handelt es sich um ein instrumentelles Handeln. Der subjektiv gemeinte Sinn des Handelns, die Intention der handelnden Person, muss nicht in jedem Fall rein zweckrational sein. Selbst wenn das illegale Handeln traditional aus purer Gewohnheit (,habit`) erfolgt (z.B. regelmäßiger Ladendiebstahl im automatisch-spontanen Entscheidungs- und Handlungsmodus) oder der Wertrationalität (z.B. Berufung zum kriminellen Wohltäter als Eigenwert statt individuelle Bereicherung) unterliegt, wäre es vermessen, es als generelles Substitut zur legalen Erwerbsarbeit (z.B. Drogenhandel, Berufskiller, organisierte Kriminalität etc.) anzusehen (Lochner 2004). Zum einen tritt Kriminalität wegen ihres Eigenwertes - z.B. aus Berufung zu „Robin Hood“ - vermutlich sehr selten auf. Meistens handelt es sich um eine Ergänzung der legalen Erwerbsarbeit (z.B. Schwarzarbeit oder Steuerhinterziehung) oder (zusätzliche oder beiläufige) Tätigkeit während der Ausübung einer beruflichen Erwerbstätigkeit (z.B. Diebstahl, Unterschlagung, Korruption oder Bilanzfälschung) (McCarthy / Hagan 2001; Piliavin / Thornton / Gartner / Matsueda 1986). Zum anderen handelt es sich um Entscheidungen unter hoher Unsicherheit, weil sowohl die Zielerreichung als auch der Ertrag im Unterschied zur legalen Erwerbsarbeit in der Regel nicht vertraglich garantiert sind (Becker / Imhof / Raimann 2007). Aber auch das affektuelle Handeln - etwa der Totschlag in Rage - kann anhand dieses Modells erklärt werden, wenn wie beim wertrationalen oder traditionalen Handeln davon ausgegangen wird, dass sich dann eine Person in einer Situation befindet, in der emotional nur noch eine einzige Handlungsalternative gesehen wird, und sie dann im Affekt automatisch-spontan statt rational kalkulierend handelt (Paternoster / Pogarsky 2009). Auch beim traditionalen Handeln von Gewohnheitsstraftätern kann gemutmaßt werden, dass eher heuristische und kognitiv routinierte Entscheidungen (,habits') als deliberierte Entscheidungen (,deliberations') getroffen werden (Wikström 2006: 79), sobald sich eine Gelegenheit für die Ausführung der Straftat ergibt (Matsueda / Kreager / Huizinga 2006).

Diese Anleihen bei der Theorie kognitiver Rationalität (Boudon 2003) und bei der ,Frame Selection Theory ' (Kroneberg / Heintze / Mehlkop 2010) können anhand der Wertrationalität legaler und illegaler Handlungen verdeutlicht werden. So geht etwa Mehlkop (2011) davon aus, dass internalisierte Normen wie etwa die kategorische Gesetzestreue, den kognitiven Handlungsset so strukturieren, dass illegale Handlungen nicht mehr kognitiv bei der subjektiven Definition der sozialen Situation und Evaluation der Handlungsalternativen (einschließlich ihrer Konsequenzen) präsent sind. Beispielsweise wäre ein Diebstahl ausgeschlossen, wenn die Norm unbedingt auferlegt ist, man dürfe nicht stehlen oder man müsse sich auf jeden Fall an Gesetze halten. Folglich handelt diese Person aus moralischer Überzeugung zumeist normkonform. ${ }^{8}$ Die Normkonformität im Sinne der Wertrationalität wiederum wird mit den gleichen Mechanismen des sozialen Handelns erklärt. Die Entsprechung der zugrundeliegenden Werte im Handeln wird durch andere Personen oder durch sich selbst belohnt (Nutzen), während der Verstoß gegen Normen entsprechende Nachteile für den Akteur (Kosten) nach sich ziehen. Gleiches gilt für traditionales Handeln: Das Einhalten von

8 In gewissen Fällen - etwa in der Situation eines moralischen Dilemmas - kann die Geltung einer solchen Norm (temporär) nicht mehr gegeben sein. Sie kann auch durch kognitive Rationalisierung „neutralisiert“ werden (Sykes / Matza 1957), indem etwa ein Versicherungsbetrug mit dem Argument, die Versicherungen seien zu gierig, legitimiert wird. Dadurch schwächen sich individuelles Normbewusstsein und Bindung des Handelns an Normen ab. 
Gewohnheiten zieht im Unterschied zum Nichteinhalten zumindest keine Kosten nach sich und tritt wegen vorteilhafter Konsequenzen eher auf als das Verwerfen von Gewohnheitsroutinen und ihrer Legitimationsgrundlage.

Solche Überzeugungen und die Bewertung von Straftaten werden der sozial-kognitiven Lerntheorie von Bandura (2001) nach durch eigene Erfahrungen oder anhand erfolgreicher Modelle in der delinquenten Bezugsgruppe gelernt (Schulz 2013). So können, so die zentrale Aussage der Theorie differentieller Assoziation (Sutherland 1968), in einer Bezugsgruppe auf der einen Seite bestimmte Straftaten geächtet werden, andere aber wiederum als akzeptabel definiert und belohnt werden (Fehr / Fischbacher 2004). Die Verfolgung eines subjektiven Sinns sowie die Ablehnung von oder die Zustimmung zu Zielen und Konsequenzen von Handlungen erfolgt wiederum nach dem Muster der subjektiv rationalen Abwägung von Vor- und Nachteilen dieses Tuns.

\section{Weitere Brückenhypothesen}

Um das Grundmodell inhaltlich zu füllen, bedarf es weiterer Brückenhypothesen zu Restriktionen und Opportunitäten. So korrelieren einerseits die Präferenzen im Allgemeinen und die Handlungsmotivation im Besonderen mit den verfügbaren Ressourcen und Gelegenheiten sowie andererseits das Investitionsrisiko mit dem Aufwand und der Erfolgswahrscheinlichkeit einer bestimmten Handlung. Beispielsweise dürfte eine Steuern hinterziehende Person versuchen, den Nutzen ihres Tuns so zu optimieren, dass das Einkommen nach Steuerabzug so hoch wie möglich bleibt. Abgesehen davon, dass eine Person nur dann die Gelegenheit für Steuerhinterziehung hat, wenn sie aufgrund des steuerpflichtigen Einkommens eine Steuererklärung abgeben muss, so sollte der Gewinn so hoch sein, dass es sich in den Augen des Akteurs lohnt. Daher liegt es nahe, dass es große Unterschiede in der Neigung für Steuerhinterziehung zwischen den Sozialschichten gibt. So bestehen für Mittel- und Oberschichten größere Anreize für Steuerhinterziehung als für einkommensschwächere Sozialschichten.

Des Weiteren muss die Person zu dieser Tat in der Lage sein, also über Wissen und Mittel für die erfolgreiche Ausführung verfügen. Eigene kognitive Fähigkeiten können hierbei genauso hilfreich sein wie etwa die Verfügbarkeit von Freunden und Bekannten, die bereits erfolgreich Steuern hinterzogen haben. Sie können bei dieser Straftat nicht nur behilflich sein, sondern auch dazu beitragen, moralische Bedenken zu zerstreuen. Ebenso kann bei den besser gebildeten, höheren Mittel- und Oberschichten nicht nur der Glaube vorherrschen, bei einer Steuerhinterziehung nicht entdeckt zu werden, sondern dass die Strafhöhen so gering seien, dass es sich generell lohnt, Steuern zu hinterziehen. Diese Überzeugung kann von delinquenten Assoziationen gelernt und belohnt werden. Folglich - und das gilt nicht nur für die Steuerhinterziehung, sondern auch für alle anderen Straftaten - tragen Kontakte mit delinquenten Gruppen zur Delinquenz bei.

Allerdings hängt der Erfolg dieses Tuns nicht nur von der Wahl der Strategie - etwa Abgabe einer falschen Steuererklärung - ab, sondern auch vom Erfolg der Gegenspieler - etwa der Steuerprüfung. So muss eine delinquente Person bei der Planung ihrer Tat die Wahrscheinlichkeit abschätzen, ob die Strafverfolgung erfolgreich ist - also in unserem Beispiel die Steuerprüfung fündig wird und eine Strafverfolgung einsetzt. Entscheidend hierbei ist die subjektiv erwartete Gewissheit und Geschwindigkeit entdeckt zu werden, während - entgegen der Abschreckungsthese in der ökonomischen Theorie der Kriminalität - die Ernsthaftigkeit der Strafe unerheblich ist (Nagin / Pogarsky 2001; Grogger 1991). Auch auf diese Einschätzung können beispielsweise erfolgreiche Steuerbetrüger unter den Freunden und Bekannten Einfluss nehmen, indem Ängste bei zögerlichen Novizen genommen werden. Andererseits zeigen Paternoster et al. (1983), dass bei Kontrolle der moralischen Verpflich- 
tung und des Zugangs zur normkonformen Bezugsgruppe die Abschreckung durch Strafe nicht gegeben ist, sondern die informelle Sanktionierung zählt.

Um eine Straftat begehen zu können, muss schließlich nicht nur die strukturelle Gelegenheit dafür bestehen, sondern es müssen auch Ressourcen (z.B. Werkzeuge oder Waffen) und (physische und psychische) Fähigkeiten (z.B. geübter Umgang mit Waffen oder Planung einer Straftat) gegeben sein. Auch hier kann ein entsprechendes Netzwerk für die Beschaffung von Informationen und Mitteln für die Straftat sowie das Erlernen der Ausführung einer Straftat von Vorteil sein.

\section{Weiteres Vorgehen}

Aufgrund der großen Variation der Opportunitäten und Restriktionen scheint es sinnvoll zu sein, in der empirischen Analyse nur bestimmte Straftaten zu fokussieren (Weisburd / Piquero 2008). Dieser Schluss kann auch dadurch begründet werden, dass es - über die Palette der Straftaten gesehen - eine erhebliche Variation gibt, dass bestimmte Gruppen überhaupt die Gelegenheit für Straftaten (Mehlkop 2011) oder die Chance für den Zugang zu delinquenten Gruppen haben (Piquero / Brezina / Turner 2005) oder dass delinquente Personen über Bezugsgruppen sanktioniert werden (Wikström 2006). Aufgrund dessen werden in der folgenden empirischen Anwendung der vorgeschlagenen Theorie häufig auftretende und daher auch besser beobachtbare Delikte wie Ladendiebstahl, Schwarzfahren, Versicherungsbetrug und Steuerhinterziehung in Betracht gezogen.

\section{Datenbasis, Variablen und statistisches Verfahren}

\section{Datenbasis}

Die Daten für die empirische Analyse basieren auf einer postalischen Befragung in Bern im Sommer 2006. Aus dem Einwohnermelderegister der Schweizer Hauptstadt wurden zufällig 4000 Adressen von erwachsenen Bürgerinnen und Bürgern im Alter zwischen 18 und 65 Jahren gezogen. Aus diesen Adressen wurden 490 Adressen für das Zwei-Phasen-Pretesting und 510 Adressen für ein Methodenexperiment zufällig ausgewählt (Becker / Mehlkop 2011). Für die Hauptuntersuchung verblieben 3000 Personen, für deren Befragung über ihre Delinquenz die ,Tailored Design Method' nach Dillman (2000) in leicht modifizierter Form angewandt wurde. Diese Personen erhielten zunächst neben dem Anschreiben, Fragebogen und frankierten Rückumschlag einen Geldbetrag von 10 Schweizer Franken als materiellen Anreiz. Abzüglich der 146 Adressen als neutrale Ausfälle (Todesfälle, Umzüge, Auslandsaufenthalt) haben sich 2230 von 2854 angeschriebenen Personen an der Studie beteiligt (78\%) und einen ausgefüllten Fragebogen zurückgeschickt. Wenn 2001 verwertbare Fragebogen in Rechnung gestellt werden, dann liegt die Ausschöpfungsquote bei 70 Prozent.

Die Daten für alltägliche Straftaten der Befragten wurden im Querschnitt erhoben. Daraus ergeben sich Einschränkungen für Interpretationen der Schätzergebnisse. Weil die Neigung für kriminelles Handeln nicht von exogenen Einflüssen, sondern von Einschätzungen des Nutzens, der Kosten und Erfolgswahrscheinlichkeiten abhängt, ist zu bedenken, dass sich diese endogenen Einflüsse in der historischen Zeit und im Lebensverlauf verändern (Mehlkop / Becker 2004: 108). Das Erlernen krimineller Fertigkeiten und Erfahrungen - das Investieren in kriminelle Spezialisierung und in spezifische kognitive Fähigkeiten für qualifikatorisch anspruchsvolle kriminelle Handlungen (z.B. Steuerhinterziehung, Betrug etc.) benötigt ebenfalls Lebenszeit (Loughran / Nguyen / Piquero / Fagan 2013: 926). So belegen Sampson und Laub $(1992,2003)$ in ihren Studien eine enorme Heterogenität des kriminellen Verhaltens im Lebensverlauf und eine bedeutsame Altersabhängigkeit bestimmter Straftaten, die wohl eher mit den Lebenslagen (etwa Ausbildung, Erwerbstätigkeit, Familie etc.) als 
mit dem Lebensalter kovariieren. Folglich wären prospektive, ereignisorientierte Längsschnittdaten die optimale Datengrundlage (Niggli 1994: 96); diese würden die Zeitveränderlichkeit kontext- und situationsspezifischer Bewertungen und Erwartungen realistischer abbilden. Für die methodisch angemessene Rekonstruktion von Kausalitäten wären solche Daten notwendig, da die subjektive Definition der sozialen Situation dem Prozess der Entscheidung für eine Handlung vorausgeht (Lauritsen 1998). In unserem Fall können wir die Problematik insofern relativieren, als ausschließlich Aussagen für die Handlungsintention in Abhängigkeit von der aktuellen sozialen Situation, den Erfahrungen im Lebensverlauf und der kriminellen Vorgeschichte gemacht werden. ${ }^{9}$

Hinsichtlich der Validität und Reliabilität werden Angaben von Befragten zu Intention und Prävalenz ihres strafbaren Handelns kritisch betrachtet (Piquero / Macintosh / Hickman 2002; Huizinga / Elliott 1986). Diverse Studien zeigen jedoch, dass die Selbstangaben hochgradig mit dem tatsächlichen Verhalten korrelieren (Pogarsky / Piquero / Paternoster 2004; Köllisch / Oberwittler 2004). Vor allem bei „Selbstausfüllern“ (z.B. postalische Befragung) werden geringe Antwortverzerrungen festgestellt (Thornberry / Krohn 2000). Dann ist auch die Neigung zu , item nonresponse ' gering (Brame / Paternoster 2003). In unserer Studie verweigerten lediglich zwei Prozent der Befragten Angaben zu früheren Straftaten und bei der Intention für Straftaten waren fehlende Angaben noch seltener. Insgesamt sind Vorbehalte gegenüber selbstberichteter Delinquenz überzogen. Angesichts der Probleme amtlicher Kriminalstatistik ist der Selbstbericht derzeit die einzige Quelle für beschreibende und ätiologische Forschung über Kriminalität und Delinquenz.

\section{Abhängige und unabhängige Variablen}

Abhängige Variablen sind delinquente Handlungen, d.h. die Neigung der Befragten für Delikte wie Ladendiebstahl, Betrug einer Hausratsversicherung, Steuerhinterziehung oder Schwarzfahrt (etwa: „Können Sie sich vorstellen, die jährliche Steuererklärung nicht wahrheitsgemäß auszufüllen, um dadurch weniger Abgaben zahlen zu müssen?"). Für jedes dieser Delikte sollten die Befragten angeben, ob sie diese straffällige Handlung nie oder doch unter Umständen begehen würden. Neben der Intention wird auch die Prävalenz von Straftaten als erklärende Variable berücksichtigt („Haben Sie selber irgendwann im Laufe Ihres Lebens eines oder mehrere der folgenden Delikte < Ladendiebstahl, Betrug einer Hausratsversicherung, Steuerhinterziehung, Schwarzfahrt > begangen?").

Dem Vorschlag von Manski (2004) folgend, werden für jede der Straftaten die Mechanismen der Entscheidung und Handlung direkt gemessen (Matsueda / Kreager / Huizinga 2006; siehe auch Tab. 1). So bemisst sich die Erfolgswahrscheinlichkeit $p$ in der subjektiv erwarteten Wahrscheinlichkeit, die jeweilige Straftat selbst erfolgreich durchführen zu können. Die Skala reicht von 1 für „sehr unwahrscheinlich“ bis 5 für „sehr wahrscheinlich“. Der materielle Ertrag E einer Straftat soll durch die befragte Person beurteilt werden, indem sie den Wert der finanziellen Einsparung auf der fünfstelligen Skala von ,sehr niedrig“ bis „sehr hoch“ einschätzen sollte. Bei der Entdeckungswahrscheinlichkeit $q$ konnten Befragte ange-

9 Um das tatsächliche strafbare Handeln aus soziologischer Perspektive für eine große Zahl von Akteuren und Handlungen empirisch zu rekonstruieren und zu erklären, wäre neben der prospektiven Messung eine retrospektive Befragung notwendig. Dieses für ökonomische Studien typische Vorgehen, bei denen Straftaten von verurteilten Straftätern in der Vergangenheit die abhängige Variable darstellen (z B. Block / Lind 1975), ist mit enormen methodischen Problemen der Rationalisierung und fehlenden Referenzgruppe normkonformer Personen behaftet (Becker / Imhof / Raimann 2007: 274). 
ben, für wie wahrscheinlich sie es halten, bei einer Straftat erwischt zu werden. ${ }^{10}$ Der Reputationsverlust $-A$ indiziert als immaterieller Ertrag, ob Befragte einen Schaden ihres Ansehens und Respekts bei der Familie oder den Freunden erleiden würden, falls sie eine bestimmte Straftat begehen. Die erwartete Strafhöhe $-C$ wurde nach Nennung der erwarteten Strafe durch die Befragten anhand folgender Frage erhoben: „Angenommen, Sie würden diese Strafe in Ihrer jetzigen Lebenssituation tatsächlich erhalten: Ist diese Strafe für Sie eher niedrig oder hoch?"11 Die Höhe wurde anhand einer Skala erfasst, die von 1 für „sehr niedrig" bis 5 für „sehr hoch“ reicht.

Die differentielle Assoziation bemisst sich zum einen danach, ob die Befragten mit Personen in ihrem Freundes- und Bekanntenkreis Kontakt haben, welche bereits Straftaten begangen haben. Zum anderen nach der Anzahl von Personen aus dem Freundes- und Bekanntenkreis, welche Steuern hinterziehen oder einen Ladendiebstahl begehen oder die Versicherung betrügen oder ohne gültiges Ticket mit öffentlichen Verkehrsmitteln fahren.

Für die normative Überzeugung bzw. die kognitiven Strategien zur Überwindung normativer Handlungsrestriktionen wird auf drei Indikatoren zurückgegriffen (Sykes / Matza 1957). Erstens wird die unbedingte Gesetzestreue der Befragten danach erfasst, inwieweit sie der Aussage zustimmen, dass man sich an Gesetze halten müsse, gleich ob man mit ihnen einverstanden oder nicht einverstanden ist. Zweitens wurden sie gebeten, eine Straftat auf einer Skala von 1 für ,überhaupt nicht schlimm“ bis 5 für ,sehr schlimm“ zu bewerten (Verneinung des Unrechts). Drittens sollten die Befragten - spezifisch für jede der vier Straftaten - die Straftat oder die Opfer dieser Straftat bewerten (Ablehnung der Straftat oder des Opfers). Für die Steuerhinterziehung wurde nach der Bewertung für die Aussage „Jeder, der nicht korrekt Steuern zahlt, schadet der Gesellschaft" gefragt. Beim Ladendiebstahl sollten die Befragten beurteilen, ob sie es als legitim ansehen, Diebstähle nur in größeren Geschäften zu begehen. Im Falle des Versicherungsbetrugs konnte die Aussage bewertet werden, Versicherungen seien gierig. Schließlich wurde gefragt, ob die öffentlichen Verkehrsbetriebe in Bern zu hohe Preise verlangen.

Die Gelegenheitsstruktur ist eine bedeutsame Voraussetzung für delinquentes Handeln. Sie wird bei der Steuerhinterziehung über die Höhe des logarithmierten Einkommens, beim Ladendiebstahl über die Häufigkeit des Einkaufens in der Berner Innenstadt oder in Einkaufszentren, Versicherungsbetrug über die Häufigkeit der früheren Schadensmeldungen

10 Da die subjektiven Entscheidungsgewichte, die Personen den Ereignissen zuschreiben, in der Regel nicht mit den tatsächlich zu erwartenden Wahrscheinlichkeiten ihres Eintretens übereinstimmen, wurden im Sinne der ,Prospect theory ' zwei unterschiedliche Messungen der Entdeckungswahrscheinlichkeiten vorgenommen. Nach Kahneman (2011: 383) ist zu erwarten, dass unwahrscheinlich erscheinende Ereignisse übergewichtet werden, vermeintlich sichere Ereignisse im Verhältnis zur tatsächlichen Eintrittswahrscheinlichkeit unterschätzt werden. Zunächst wurden die Befragten gebeten, die Entdeckungswahrscheinlichkeit auf einer Skala - reichend von 1 (sehr unwahrscheinlich) bis 5 (sehr wahrscheinlich) - anzugeben. Dann sollten sie angeben, in wie vielen Fällen von 100 die Straftat entdeckt wird. Diese beiden Entdeckungswahrscheinlichkeiten korrelieren miteinander. Der Korrelationskoeffizient für Steuerhinterziehung, Ladendiebstahl und Versicherungsbetrug beträgt jeweils 0,4 und für Schwarzfahren 0,5 .

11 Beim Ladendiebstahl wurde die erwartete Strafe mit folgender Frage erhoben: „Nehmen wir einmal an, Sie würden in einem Kaufhaus Waren im Wert von 250 Franken mitnehmen, ohne dafür zu bezahlen. Welche Strafe erhalten Sie Ihrer Meinung nach, wenn Sie zum ersten Mal bei solch einem Ladendiebstahl erwischt werden? Wählen Sie bitte nur eine der folgenden Antwortmöglichkeiten aus.“. Die vorgegebenen Antworten waren: Verwarnung und/oder Hausverbot, sonst keine Folgen; geringe Geldstrafe und eine Anzeige; hohe Geldstrafe und eine Anzeige; Haft auf Bewährung. 
und beim Schwarzfahren über den Besitz eines Führerscheins (für ein Kraftfahrzeug) erfasst. ${ }^{12}$ Zudem wird bei der Steuerhinterziehung die Klassenlage der Befragten kontrolliert. Aufgrund der geringen Anteile von Arbeitern und Angehörigen der oberen Dienstklasse wird das zugrunde gelegte Klassenschema von Erikson und Goldthorpe (1992) auf drei soziale Klassen - die Unter- bzw. Arbeiterschichten, die Mittelschichten und die obere Mittelund Oberschicht - zusammengefasst.

Tabelle 1: Übersicht über die Operationalisierung der Variablen am Beispiel der Steuerhinterziehung

\begin{tabular}{|c|c|}
\hline Abhängige Variable & Originalfrage \\
\hline Steuerhinterziehung & $\begin{array}{l}\text { Können Sie sich vorstellen, die jährliche Steuererklärung nicht } \\
\text { wahrheitsgemäß auszufüllen, um dadurch weniger Abgaben zahlen } \\
\text { zu müssen? (Antwort: ja/nein) }\end{array}$ \\
\hline \multicolumn{2}{|l|}{ Unabhängige Variablen } \\
\hline Erfolgswahrscheinlichkeit $\mathrm{p}$ & $\begin{array}{l}\text { Steuerhinterziehung: Für viele Verhaltensweisen benötigt man Ta- } \\
\text { lent und Geschicklichkeit. Für wie wahrscheinlich halten Sie es, } \\
\text { dass Sie selbst unentdeckt Steuern hinterziehen können? (Antwor- } \\
\text { ten von } 1 \text { für sehr unwahrscheinlich bis } 5 \text { für sehr wahrscheinlich) }\end{array}$ \\
\hline Materieller Ertrag E & $\begin{array}{l}\text { Stellen Sie sich vor, Sie machen falsche Angaben bei der Steuerer- } \\
\text { klärung und sparen dadurch } 2000 \text { Franken ein. Wie hoch schätzen } \\
\text { Sie persönlich den Wert dieser Einsparung ein? (Antworten von } 1 \\
\text { für sehr niedrig bis } 5 \text { für sehr hoch) }\end{array}$ \\
\hline Entdeckungswahrscheinlichkeit q & $\begin{array}{l}\text { Wieder angenommen, Sie füllen Ihre jährliche Steuererklärung } \\
\text { nicht wahrheitsgetreu aus. Wie wahrscheinlich ist es Ihrer Ansicht } \\
\text { nach, dass man Sie dabei erwischt? (Antworten von } 1 \text { für sehr un- } \\
\text { wahrscheinlich bis } 5 \text { für sehr wahrscheinlich) }\end{array}$ \\
\hline Reputationsverlust $-\mathrm{A}$ & $\begin{array}{l}\text { Denken Sie, dass Sie bei Ihren besten Freunden und Ihrer Familie } \\
\text { an Respekt und Ansehen verlieren würden, wenn Ihre Freunde oder } \\
\text { Familie wüssten, dass Sie Steuern hinterziehen? (Antwort: ja/nein) }\end{array}$ \\
\hline Strafhöhe $-\mathrm{C}$ & $\begin{array}{l}\text { (Nehmen wir einmal an, Sie füllen Ihre jährliche Steuererklärung } \\
\text { nicht wahrheitsgetreu aus. Welche Strafe würden Sie dafür erhal- } \\
\text { ten?) Angenommen, Sie würden diese Strafe in Ihrer jetzigen Le- } \\
\text { benssituation tatsächlich erhalten: Ist diese Strafe für Sie eher nied- } \\
\text { rig oder eher hoch? (Antwort von } 1 \text { für sehr niedrig bis } 5 \text { für sehr } \\
\text { hoch) }\end{array}$ \\
\hline $\begin{array}{l}\text { Freunde/Bekannte mit } \\
\text { Steuerbetrug }\end{array}$ & $\begin{array}{l}\text { Gibt es in Ihrem Bekanntenkreis Personen, von denen Sie wissen, } \\
\text { dass sie ab und zu in der Steuererklärung falsche Angaben machen? } \\
\text { (Antwort: ja/nein) }\end{array}$ \\
\hline Anzahl dieser Personen & $\begin{array}{l}\text { Schätzen Sie bitte, wie viele Personen aus Ihrem Freundes- und Be- } \\
\text { kanntenkreis ihre Steuererklärung ab und zu nicht korrekt ausfüllen, } \\
\text { um dadurch Abgaben einzusparen. (Antwortkategorien: } 1 \text { für } 0 \\
\text { bis } 7 \text { für 9-10 Personen) }\end{array}$ \\
\hline Unbedingte Gesetzestreue & $\begin{array}{l}\text { Im Folgenden finden Sie eine Reihe von Aussagen zur Gesellschaft. } \\
\text { Wir würden gerne wissen, ob Sie diesen Aussagen zustimmen oder } \\
\text { ob Sie diese Aussagen ablehnen. An Gesetze muss man sich halten, } \\
\text { egal ob man mit ihnen einverstanden oder nicht einverstanden ist. } \\
\text { (Antworten von } 1 \text { für stimme überhaupt nicht zu bis } 5 \text { für stimme } \\
\text { stark zu) }\end{array}$ \\
\hline
\end{tabular}

12 Dieser Indikator ist allgemeiner als der für den Besitz eines KFZ, da mit dem Führerschein nicht nur eigene, sondern auch Fahrzeuge anderer Besitzer (Freunde, Autovermietung, car sharing) gelenkt werden können. 


\begin{tabular}{l|l}
\hline Bewertung der Straftat & $\begin{array}{l}\text { Im Folgenden sind vier Delikte genannt. Bitte sagen Sie bei jedem } \\
\text { Delikt, für wie schlimm Sie es halten. Für wie schlimm halten Sie } \\
\text { Steuerhinterziehung? (Antwort von 1 für überhaupt nicht schlimm } \\
\text { bis 5 für sehr schlimm) }\end{array}$ \\
\hline Bewertung der Straftäter & $\begin{array}{l}\text { Wie ist Ihre Meinung zur folgenden Aussage? Jeder, der nicht kor- } \\
\text { rekt Steuern zahlt, schadet der Gesellschaft. (Antworten von 1 für } \\
\text { stimme überhaupt nicht zu bis 5 für stimme stark zu) }\end{array}$ \\
$\begin{array}{l}\text { Ökonomische Integrität } \\
\text { Soziale Anerkennung }\end{array}$ & $\begin{array}{l}\text { Faktorenlösung aus folgenden Items: } \\
\text { Wie wichtig ist es Ihrer Meinung nach, hart zu arbeiten, ein sicheres } \\
\text { und regelmäßiges Einkommen zu haben, den Lebensstandard zu er- } \\
\text { halten, sich wohl zu fühlen, geliebt und geschätzt zu werden? (Ant- } \\
\text { worten von 1 für überhaupt nicht wichtig bis 5 für sehr wichtig) } \\
\text { Wir würden gerne wissen, ob Sie diesen Aussagen zustimmen oder } \\
\text { ob Sie diese Aussagen ablehnen. In unserer Gesellschaft muss jede } \\
\text { und jeder zum Allgemeinwohl beitragen. Das Eigentum anderer } \\
\text { Menschen muss geachtet werden. Bereicherung auf Kosten anderer } \\
\text { Bürgerinnen und Bürger ist verwerflich. (Antworten von 1 für stim- } \\
\text { me überhaupt nicht zu bis 5 für stimme stark zu) } \\
\text { Für wie wichtig halten Sie persönlich diese Merkmale für die eige- } \\
\text { ne berufliche Tätigkeit? (Bitte beantworten Sie diese Frage auch, } \\
\text { wenn Sie momentan keiner beruflichen Tätigkeit nachgehen). Ho- } \\
\text { hes Einkommen? Ein Beruf, der für die Gesellschaft nützlich ist? } \\
\text { Gute Aufstiegsmöglichkeiten? (Antworten von 1 für überhaupt } \\
\text { nicht wichtig bis 5 für sehr wichtig) }\end{array}$ \\
\hline
\end{tabular}

Am Beispiel für die Steuerhinterziehung, die im Zentrum der empirischen Analyse steht, werden die Items für die individuellen Bewertungen und Erwartungen im Wortlaut dargestellt. Für die anderen Delikte sind sie ähnlich und werden aus Platzgründen und Redundanz nicht explizit dargestellt.

\section{Statistisches Verfahren}

Für multivariate Analysen wird die binäre logistische Regression angewandt. Neben einer verteilungstheoretischen kann auch eine entscheidungstheoretische Begründung angeführt werden (Urban 1993: 108). Wie zuvor gesehen, wird die Delinquenz als soziales Handeln mit einem handlungstheoretischen Ansatz der subjektiven Werterwartung, der subjektiv rationalen Wahl von Handlungsalternativen, theoretisch modelliert. Mithilfe der logistischen Regression kann diese Logik des sozialen Handelns statistisch modelliert werden.

Für die Intention eine Straftat zu begehen werden die ,Average Marginal Effects ' (AME) als Maßzahl für die „,mittlere“ Stärke des Effekts auf die Wahrscheinlichkeit eine Straftat zu begehen geschätzt. Letztere erlauben den Vergleich der Koeffizienten über (verschachtelte) Modellierungen hinweg und minimieren Verzerrungen, die sich aus der Problematik unbeobachteter Heterogenität ergeben (Hinz / Auspurg 2011).

\section{Empirische Befunde}

\section{Beschreibung der Massendelikte als soziales Handeln}

Im theoretischen Teil ist in Anlehnung an Merton (1949) davon ausgegangen worden, dass allgemein akzeptierte und daher legitime gesellschaftliche Ziele und Werte - wie etwa ökonomischer Wohlstand, soziale Anerkennung, Beitrag zum Allgemeinwohl oder Achtung der Eigentumsrechte anderer - einen besonderen Stellenwert für die individuelle Auswahl strafbarer oder legaler Handlungen als Mittel für die Erreichung dieser Ziele bzw. Erfüllung dieser Werte spielen. So stimmt mehr als die Hälfte der Befragten uneingeschränkt zu, dass je- 
de Bürgerin und jeder Bürger zum Allgemeinwohl beitragen solle, dass das Eigentum anderer zu respektieren sei und dass die Bereicherung auf Kosten anderer Bürgerinnen und Bürger verwerflich sei (Abb. 2). Fast die Hälfte der Befragten findet es richtig, dass man hart arbeiten oder den Lebensstandard selbständig erhalten soll. Während fast die Hälfte ein hohes Einkommen als wichtig erachtet, finden rund 92 Prozent der Befragten, ein sicheres und regelmäßiges Einkommen sei bedeutsam. Soziale Anerkennung - d.h. geliebt und geschätzt werden, einen für die Gesellschaft nützlichen Beruf oder einen anerkannten und geachteten Beruf auszuüben - ist für die Mehrheit der Befragten ebenfalls besonders wichtig. Insgesamt werden in der Tat die theoretisch angenommenen Ziele, Werte und Mittel mehrheitlich als legitim angesehen.

Abbildung 2: Zustimmung zu kulturellen und sozialen Werten und Zielen (in Prozent)

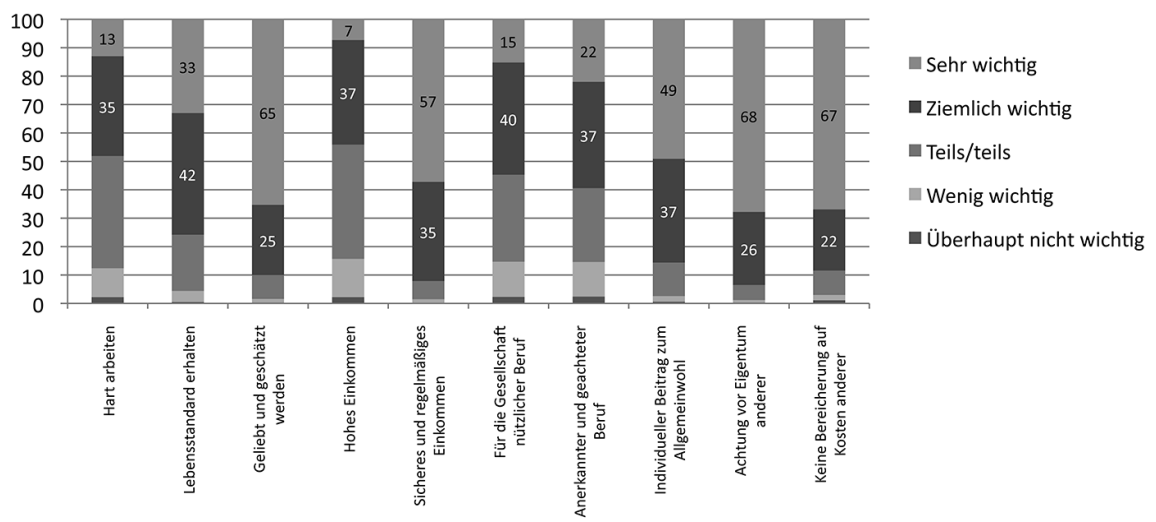

Wie bewerten die Befragten aber Straftaten wie etwa Ladendiebstahl, Steuerhinterziehung, Versicherungsbetrug oder Schwarzfahren? Alle Straftaten werden mit Ausnahme der Nutzung öffentlicher Verkehrsmittel ohne gültiges Ticket überwiegend geächtet (Abb. 3).

Abbildung 3: Bewertung der Straftaten - Prozentuale Verteilung

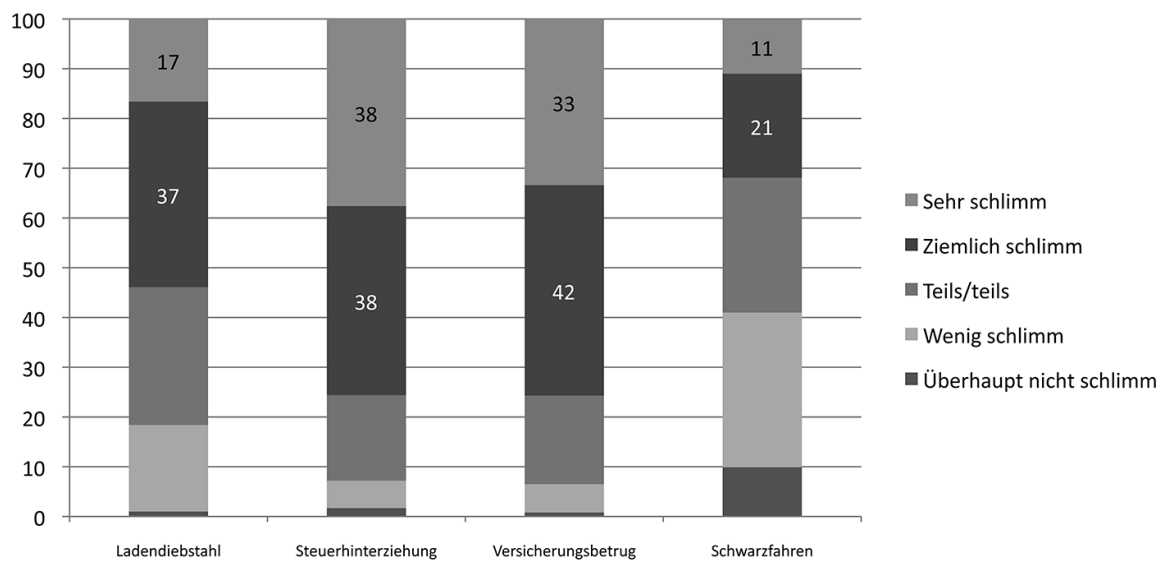


Wie verteilen sich Intention und Prävalenz von Straftaten unter den Befragten? So geben fast zwei Drittel der Befragten zu, in der Vergangenheit ohne gültiges Ticket die öffentlichen Verkehrsmittel in Bern benutzt zu haben, und 46 Prozent beabsichtigen auch in $\mathrm{Zu}-$ kunft, schwarzzufahren (Abb. 4). Hingegen berichten fünf Prozent der Befragten, dass sie in der Vergangenheit Steuern hinterzogen haben. Obgleich die Steuerhinterziehung am häufigsten als besonders verwerflich angesehen wird, sind rund ein Viertel der Befragten unter Umständen bereit Steuern zu hinterziehen. Diese Intention ist deutlich höher als für Ladendiebstahl und geringfügig höher als für einen beabsichtigten Versicherungsbetrug. Aufgrund dieses augenfälligen Zusammenhangs von moralischer Bewertung und Intention bzw. Prävalenz wird die Steuerhinterziehung in einem weiteren Analyseschritt detaillierter betrachtet. ${ }^{13}$

\section{Abbildung 4: Intention und Prävalenz für Massendelikte - Prozentuale Verteilung}

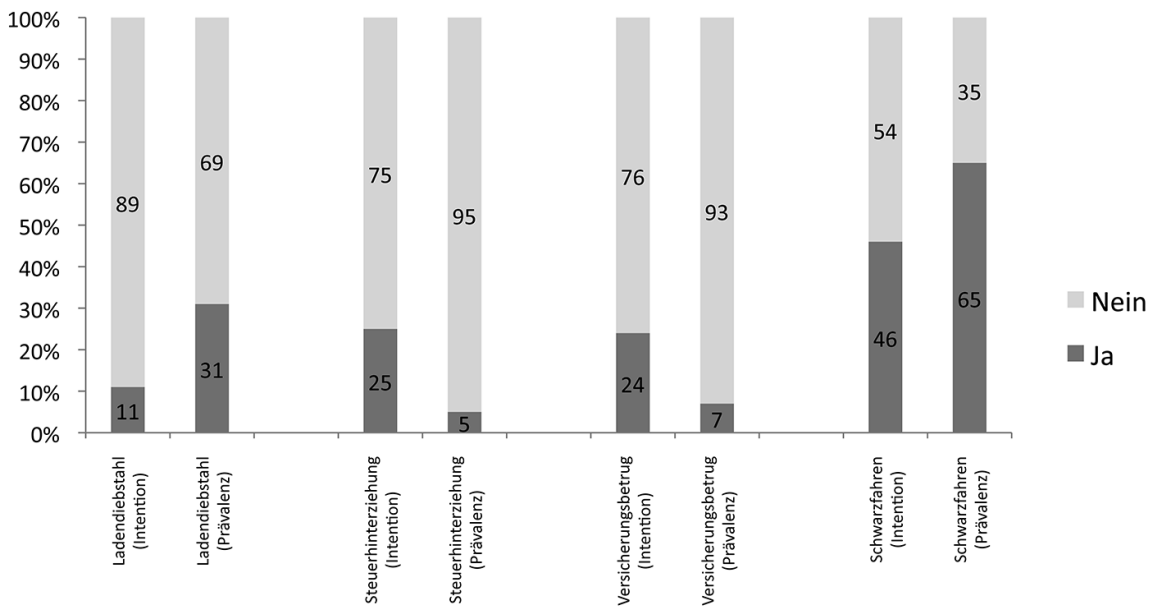

Aus entscheidungs- und handlungstheoretischer Perspektive ist die Steuerhinterziehung besonders interessant - zumal aus ökonomischer Sicht diesem Handeln in der Regel ein rein zweckrationaler Sinn und eine rational kalkulierte Entscheidung unterstellt wird. Unseren Daten zufolge hat sich aber mehr als ein Drittel der Befragten noch nie Gedanken über Vorteile dieser Straftat gemacht (Tab. 2). Genauso viele Befragten wissen offensichtlich nicht, was sie bei der Steuererklärung tun müssten, um sich Vorteile zu verschaffen. Rund 18 Prozent meinen, dass es sich für sie nicht lohne, Steuern zu hinterziehen, da sie ohnehin nur geringe Steuern zahlen; der geringste Anteil von fast 10 Prozent ist vom (egoistischen) Nutzen dieser Straftat überzeugt.

13 Die detaillierte Analyse dieser Straftat ist zudem deswegen interessant, weil fast drei Viertel der Befragten dem ,free riding 'bei der Steuerzahlung (,Solange die anderen Leute Steuern zahlen, spielt es keine Rolle, wenn ich keine Steuern zahle") überhaupt nicht zustimmen können. Vielmehr sind sich 83 Prozent der Befragten darin einig, dass jeder, der nicht korrekt Steuern zahlt, auch der Gesellschaft schadet. 
Tabelle 2: Steuerhinterziehung - Rein zweckrationale Abwägung von Kosten und Nutzen?

Frage: Welche der folgenden Aussagen trifft am ehesten auf Sie persönlich zu? Bitte geben Sie nur eine Antwort an.

Über den Vorteil habe ich mir noch nie Gedanken ge-
macht.

Falsche Angaben lohnen sich nicht, da ich nur wenig Steuern zahle.

Ich weiß nicht, welche Angaben ich verändern müsste, um Geld zu sparen.

Wenn ich falsche Angaben machen würde, könnte ich viel Geld sparen.

Insgesamt

(in Klammern: n)

\begin{tabular}{|c|ccc}
$\begin{array}{c}\text { Alle Be- } \\
\text { fragten }\end{array}$ & $\begin{array}{c}\text { Unter- } \\
\text { und Ar- } \\
\text { beiter- } \\
\text { schich- } \\
\text { ten }\end{array}$ & $\begin{array}{c}\text { Mittel- } \\
\text { schich- } \\
\text { ten }\end{array}$ & $\begin{array}{c}\text { Obere } \\
\text { Mittel- } \\
\text { und } \\
\text { Ober- } \\
\text { schicht }\end{array}$ \\
\hline $36,3 \%$ & $39,7 \%$ & $35,1 \%$ & $33,8 \%$ \\
$17,6 \%$ & $17,1 \%$ & $18,2 \%$ & $17,8 \%$ \\
$36,5 \%$ & $40,8 \%$ & $39,0 \%$ & $30,4 \%$ \\
$9,6 \%$ & $2,4 \%$ & $7,7 \%$ & $18,0 \%$ \\
\hline $\begin{array}{c}100,0 \% \\
(1.957)\end{array}$ & $\begin{array}{c}100,0 \% \\
(468)\end{array}$ & $\begin{array}{c}100,0 \% \\
(818)\end{array}$ & $\begin{array}{c}100,0 \% \\
(601)\end{array}$ \\
\hline
\end{tabular}

Diese Einschätzungen variieren - mit Ausnahme für den fehlenden Nutzen - zwischen den Sozialschichten. Angehörige der mittleren und höheren Sozialschichten haben eher über die Vorteile einer Steuerhinterziehung nachgedacht als Angehörige der unteren Sozialschichten, die im Vergleich zur höheren Schicht nicht wissen, welche Angaben in der Steuererklärung sie ändern müssten, um Gewinne zu erzielen. Für sie besteht im Unterschied zur oberen Mittel- und Oberschicht auch kein Anreiz hierfür. Denn während rund zwei Prozent der Arbeiter glauben, viel Geld sparen zu können, ist dies für acht Prozent der Personen in den Mittelschichten und für 18 Prozent aus den höheren Schichten der Fall.

Tabelle 3: Steuerhinterziehung - Erwartete Strafe

\begin{tabular}{|c|c|c|c|c|}
\hline $\begin{array}{l}\text { Frage: Nehmen wir einmal an, Sie füllen Ihre jährliche } \\
\text { Steuererklärung nicht wahrheitsgetreu aus. Welche Strafe } \\
\text { würden Sie dafür erhalten? Kreuzen Sie bitte die von Ih- } \\
\text { nen erwartete Strafe an. }\end{array}$ & $\begin{array}{c}\text { Alle } \\
\text { Befrag- } \\
\text { ten }\end{array}$ & $\begin{array}{l}\text { Unter- } \\
\text { und Ar- } \\
\text { beiter- } \\
\text { schich- } \\
\text { ten }\end{array}$ & $\begin{array}{l}\text { Mittel- } \\
\text { schich- } \\
\text { ten }\end{array}$ & $\begin{array}{l}\text { Obere } \\
\text { Mittel- } \\
\text { und } \\
\text { Ober- } \\
\text { schicht }\end{array}$ \\
\hline Das hat keine Konsequenzen. & $2,0 \%$ & $2,6 \%$ & $2,0 \%$ & $2,0 \%$ \\
\hline Man muss die fehlenden Steuerabgaben nachzahlen. & $25,3 \%$ & $28,8 \%$ & $26,1 \%$ & $21,3 \%$ \\
\hline $\begin{array}{l}\text { Man muss fehlende Steuerabgaben nachzahlen und erhält } \\
\text { eine geringe Busse. }\end{array}$ & $38,7 \%$ & $35,5 \%$ & $39,3 \%$ & $40,8 \%$ \\
\hline $\begin{array}{l}\text { Man muss mit einem Strafverfahren wegen Steuerhinter- } \\
\text { ziehung rechnen. }\end{array}$ & $34,0 \%$ & $33,1 \%$ & $32,6 \%$ & $36,0 \%$ \\
\hline $\begin{array}{l}\text { Insgesamt } \\
\text { (in Klammern: } \mathrm{n} \text { ) }\end{array}$ & $\begin{array}{l}100,0 \% \\
(1.957)\end{array}$ & $\begin{array}{l}100,0 \% \\
(468)\end{array}$ & $\begin{array}{c}100,0 \% \\
(818)\end{array}$ & $\begin{array}{c}100,0 \% \\
(601)\end{array}$ \\
\hline
\end{tabular}

Diesen Einschätzungen stehen folgende Erwartungen für Strafen gegenüber (Tab. 3). Ein verschwindend geringer Anteil der Befragten geht davon aus, die Steuerhinterziehung hätte keinerlei Konsequenzen für sie. Fast drei Viertel der Befragten kennen die objektiv zutreffende Strafe (Nachzahlung, Buße und Strafverfahren). Für diese Erwartung gibt es keine bedeutsamen Unterschiede nach Schichtzugehörigkeit, wobei die höheren Sozialschichten etwas besser über die tatsächlichen Strafmaße informiert zu sein scheinen. 


\section{Multivariate Analysen}

Im Folgenden werden die zentralen Argumente der strukturell-individualistischen Erklärung delinquenten Handelns - zunächst anhand der Neigung für Steuerhinterziehung - schrittweise überprüft (Tab. 4). In der Tat nehmen Individuen eine subjektiv rationale Abwägung der Kosten und Nutzen von Handlungsalternativen vor.

Je höher die subjektiv erwartete Erfolgswahrscheinlichkeit und je höher der subjektiv erwartete materielle Ertrag einer Steuerhinterziehung sind, desto eher wird diese Straftat bei einer anstehenden Steuererklärung erwogen. Je höher die Entdeckungswahrscheinlichkeit, je höher der Verlust von Prestige und Ansehen und je höher die Strafe erscheinen, desto eher wird von einer Steuerhinterziehung abgesehen (Modell 1). Entsprechend der, Prospect Theory 'ist der Effekt der Entdeckungswahrscheinlichkeit q und des befürchteten Reputationsverlusts -A größer als die Erfolgswahrscheinlichkeit $\mathrm{p}$ und der materielle Gewinn E, weil Individuen eher Verluste zu vermeiden versuchen als in riskanter Weise zusätzliche Gewinne zu erzielen. Kurzum: Je höher die Handlungsmotivation und je geringer das Investitionsrisiko sind, desto eher ist eine Person geneigt Steuern zu hinterziehen (Modell 2). Betrachten wir das multiplikative Modell (3): Je höher für eine Person der subjektiv erwartete Nettonutzen einer Steuerhinterziehung gegenüber dem SEU-Wert anderer Alternativen erscheint, desto eher ist sie versucht, unwahre Angaben bei der Steuererklärung zu machen.

Die differentielle Assoziation hat den theoretisch erwarteten Einfluss (Modell 4): Befinden sich Freunde und Bekannte im Netzwerk einer Person, die ebenfalls Steuern hinterziehen, dann steigt die Neigung selbst Steuern zu hinterziehen. Je größer die Anzahl der Steuern hinterziehenden Freunde und Bekannte ist, desto eher ist die Fokusperson geneigt ebenfalls Steuern zu hinterziehen. ${ }^{14}$

Werden in einem weiteren Schritt normative Überzeugungen wie etwa die kategorische Gesetzestreue, die Verwerflichkeit von Steuerhinterziehung als normative Bewertung der Straftat und die Bewertung der Straftäter als unsoziale Personen, die der Gesellschaft einen Schaden zufügen, ins Spiel gebracht, dann ist ein unerwarteter Befund festzustellen (Modell 5). Entgegen den Befunden von Kroneberg / Heintze / Mehlkop (2010) oder Becker / Mehlkop (2006) hat die Gesetzestreue keinen statistisch signifikanten Effekt auf die Intention unter Umständen Steuern zu hinterziehen, wenn weitere Einflüsse kontrolliert werden. Werden Straftat und Täter subjektiv geächtet, dann wird eine Steuerhinterziehung nicht erwogen. Offensichtlich korreliert diese moralische Dimension mit einer Unterlassung von Steuerhinterziehung.

14 Theoretisch konsistent korreliert bei einer Steuerhinterziehung die differentielle Assoziation negativ mit dem erwarteten Reputationsverlust bei Familie und Freunden (Kontakte: -0,139; Menge der Kontakte: -0,165). 


\begin{tabular}{|c|c|c|c|c|c|}
\hline$a$ & 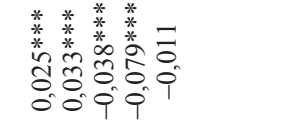 & \begin{tabular}{ll}
$*$ & $*$ \\
$*$ & $*$ \\
$*$ & $*$ \\
\multirow{2}{*}{} & \multirow{2}{*}{} \\
0 & 0 \\
0 & 0
\end{tabular} & 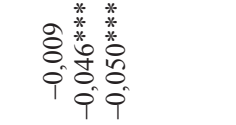 & 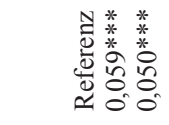 & 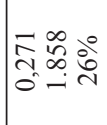 \\
\hline$\infty$ & 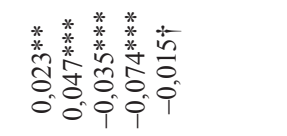 & 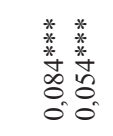 & 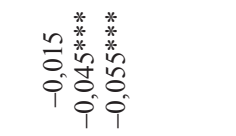 & $\begin{array}{l}* \\
\stackrel{*}{m} \\
0\end{array}$ & 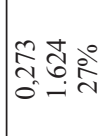 \\
\hline$r$ & 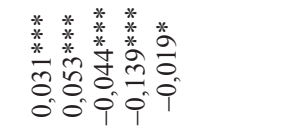 & 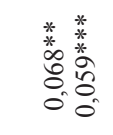 & 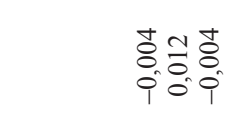 & 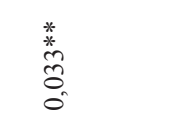 & 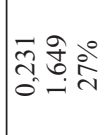 \\
\hline 6 & & & 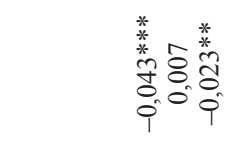 & & 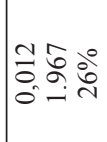 \\
\hline in & 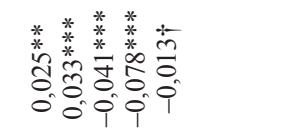 & 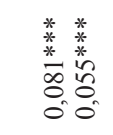 & 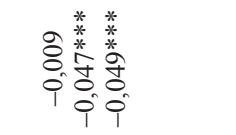 & & 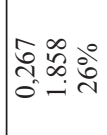 \\
\hline$\nabla$ & 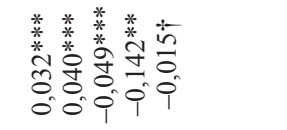 & $\begin{array}{ll}* & * \\
* & * \\
* & * \\
0 & * \\
0 & 0 \\
0 & 0 \\
0 & 0\end{array}$ & & & 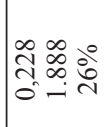 \\
\hline$m$ & 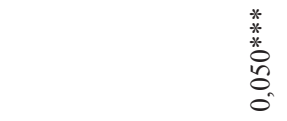 & & & & 莳京 \\
\hline$\sim$ & \begin{tabular}{ll}
$*$ & $*$ \\
$*$ & $*$ \\
$*$ & $*$ \\
\multirow{5}{*}{} & 0 \\
0 & 0 \\
0 & 0
\end{tabular} & & & & \begin{tabular}{l}
$m$ \\
$\stackrel{9}{\ddagger}$ \\
\hdashline
\end{tabular} \\
\hline- & 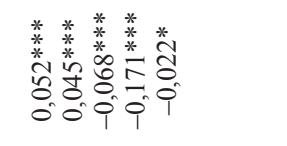 & & & & 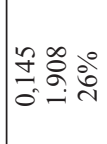 \\
\hline$\frac{0}{\stackrel{0}{0}}$ & 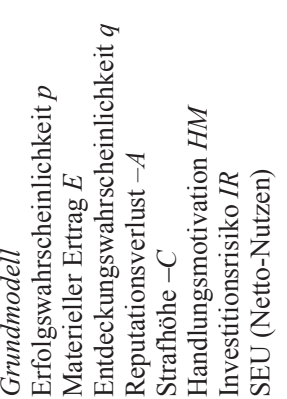 & 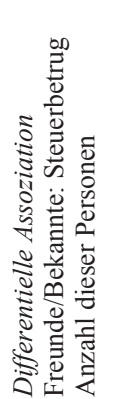 & 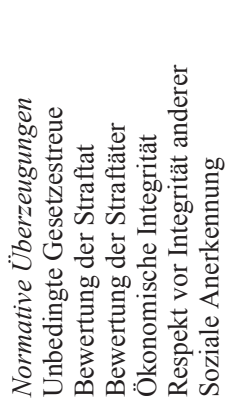 & 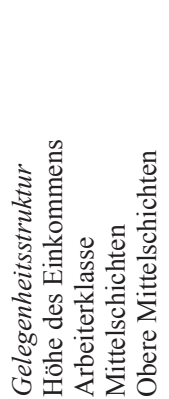 & 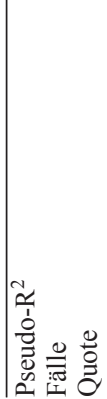 \\
\hline
\end{tabular}


Werden gesellschaftliche Ziele und Werte wie etwa das Streben nach ökonomischer Integrität oder sozialer Anerkennung sowie der Respekt der Eigentumsrechte anderer kontrolliert, so beeinflussen eher die individualistischen Ziele die Entscheidung (Modell 6). Werden jedoch die Mechanismen der Handlungsentscheidung, der differentiellen Kontakte und der Gelegenheitsstruktur (bemessen anhand der Höhe des individuellen Einkommens), dann sind die Effekte dieser normativen Dimensionen nicht mehr statistisch signifikant (Modell 7). Jedoch gibt es ein Wechselspiel im Sinne der Theorie differentieller Assoziation, dass Befragte, die Steuern hinterziehende Personen in ihrem Bekannten- und Freundeskreis haben, sowohl eine kategorische Gesetzestreue ablehnen als auch die Steuerhinterziehung nicht als verwerfliche Straftat erachten. Sie befürchten in dem Fall auch keinen Verlust an Respekt und Ansehen, sondern gehen davon aus, über Steuerhinterziehung bei ihnen an sozialer Anerkennung zu gewinnen.

Hingegen bleiben die Neutralisierungseffekte durch Ächtung der Straftat und der Täter (Modell 5) statistisch signifikant, wenn die Gelegenheitsstruktur kontrolliert wird: Je höher das eigene zu versteuernde Einkommen ist, desto größer ist der Anreiz Steuern zu hinterziehen, um entsprechende Nutzenbeträge zu sichern (Modell 8). Es gibt „gute Gründe“ dafür zu vermuten, dass die Gelegenheitsstruktur für Steuerhinterziehung durch die Struktur sozialer Klassen verläuft (Becker / Mehlkop 2006). Wie nicht anders zu erwarten war, neigen Angehörige der Mittel- und Oberschichten eher dazu Steuern zu hinterziehen als Personen aus den Unter- und Arbeiterschichten (Modell 9).

Tabelle 5: Korrelation sozialer Mechanismen der Steuerhinterziehung mit Schichtzugehörigkeit

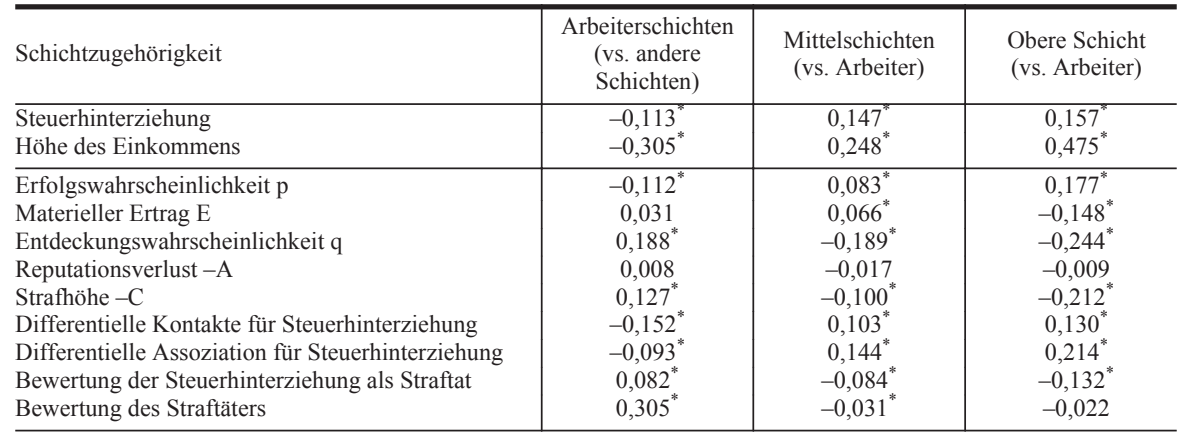

$* \mathrm{p} \leq 0,01$

Diese Schichtunterschiede lassen sich durch die schichttypischen Definitionen der sozialen Situation (hier: Gelegenheitsstruktur und Lebenslage), Evaluationen von Vor- und Nachteilen einer Steuerhinterziehung, Erfolgs- und Entdeckungswahrscheinlichkeiten, differentielle Assoziation und Bewertung der Straftat plausibilisieren (Tab. 5). ${ }^{15}$ Ohne ins Detail zu gehen, zeigen sich systematische, theoriekonsistente Unterschiede zwischen den Arbeitern und anderen Sozialschichten. Arbeiter glauben, weniger erfolgreich bei der Steuerhinterziehung zu sein und auch eher entdeckt zu werden. In der Einschätzung des materiellen Nutzens unterscheiden sie sich nicht von den anderen Sozialschichten. Aber der Betrag des Gewinns ist

15 Weil Interaktionsterme in logistischen Regressionen nicht interpretierbar sind, werden in der Logik des Modells 9 in Tabelle 3 die Korrelationskoeffizienten (Pearson) für den Zusammenhang von Klassenlage und Mechanismen der Steuerhinterziehung für die einzelnen Sozialschichten betrachtet. 
für die Mittelschichten höher als für Arbeiter, während die oberen Schichten die Einsparung von Steuerzahlungen geringer als Arbeiter beurteilen. Der subjektiv erwartete Verlust der Reputation allerdings korreliert nicht mit der Klassenlage. Arbeiter schätzen die Strafmaße höher ein und verfügen seltener über die delinquenten differentiellen Kontakte. Schließlich verurteilen sie die Steuerhinterziehung und die Täter eher - möglicherweise aufgrund unterschiedlicher Lebensführung - als Angehörige der mittleren und höheren Sozialschichten. ${ }^{16}$

Zum Abschluss werden die Mechanismen aller vier Straftaten im Vergleich analysiert (Tab. 6). Was die subjektiv erwarteten Erfolgs- und Entdeckungswahrscheinlichkeiten sowie die materiellen und immateriellen Erträge anbelangt, entsprechen die Schätzergebnisse jeweils für alle Straftaten den theoretischen Annahmen - mit Ausnahme für die subjektiv erwarteten Strafmaße, die den rigiden ökonomischen Modellen zufolge als Abschreckungsfaktor gelten. Einerseits haben sie in allen Grundmodellen (1) das geringste Gewicht. Das bedeutet, dass ihnen im Vergleich zu den anderen Werten und Erwartungen bei der Handlungsentscheidung eine relativ geringe Bedeutung zukommt. Andererseits sind sie bei den Straftaten, die in der Strafverfolgung selbst mit vergleichsweise geringen Strafmaßen einhergehen, statistisch insignifikant. Weil die subjektiv erwarteten Wahrscheinlichkeiten entdeckt $\mathrm{zu}$ werden generell statistisch und in der Höhe bedeutsam sind, werden hiermit die Studien bestätigt, welche zum Schluss gelangen, dass nicht die Strafhöhe, sondern die Gewissheit einer Entdeckung und Bestrafung eine abschreckende Wirkung hat. ${ }^{17}$ Für alle Straftaten haben Erfolgswahrscheinlichkeit und materielle Erträge einen geringeren Einfluss auf die Intention als die Entdeckungswahrscheinlichkeit und der Reputationsverlust.

Bei allen Straftaten hat die differentielle Assoziation den theoretisch erwarteten Einfluss (Modelle 2). ${ }^{18}$ Die Delinquenz steigt mit der Anzahl delinquenter Personen im sozialen Netzwerk. Weiterführende, hier nicht dokumentierte Korrelationsanalysen belegen für alle Straftaten, dass die diese Kontakte - wie von der Theorie differentieller Assoziation prognostiziert - mit der Ablehnung kategorischer Gesetzestreue und dem erwarteten Gewinn an

16 Zudem zeigen weiterführende, hier nicht dokumentierte Analysen, dass die Angehörigen aus oberen Sozialschichten gerade dann eine Steuerhinterziehung als nicht oder wenig schlimme Tat ansehen und die Täter auch nicht verachten, wenn sie selbst in der Vergangenheit Steuern hinterzogen haben. Ob hierbei eine Kausalität im Sinne der Effekte differentieller Assoziation oder Effekte kognitiver Dissonanz (Neutralisierung) vorliegen, kann erst anhand von Längsschnittdaten abschlieBend beurteilt werden.

17 So ist die subjektiv erwartete Wahrscheinlichkeit, bei der Fahrt mit öffentlichen Verkehrsmittel (ÖV) in Bern ohne gültiges Ticket erwischt zu werden, am geringsten im Vergleich zu den anderen Straftaten. Auf einem Wertebereich von 1 bis 5 liegt der Mittelwert für Ladendiebstahl bei 3,6, für Steuerhinterziehung bei 3,5, für Versicherungsbetrug bei 3,2 und schließlich für Schwarzfahren bei 2,9. Bei Schwarzfahren glauben die Befragten, dass drei von 1000 Personen erwischt werden. Den Angaben von Bern Mobil (Betreiber der ÖV) wurden im Jahre 2007 rund 24 von 1000 und im Jahre 2012 rund 27 von 1000 kontrollierten Personen erwischt, wobei fast ein Viertel der erwischten Personen keine echten Schwarzfahrer waren, da sie lediglich ihre Abonnement-Karte nicht bei sich führten. Während 2007 noch jeder zwölfte Passagier kontrolliert wurde, wurde im Jahre 2012 jeder neunte Fahrgast kontrolliert. Das mittlere Risiko, in Bern ohne gültiges Ticket in öffentlichen Verkehrsmittel erwischt zu werden, liegt nach Auskunft von Bern Mobil bei eins zu 112 (für Details: http://blog.derbund.ch/datenblog/index.php/ 1334/bern-zuerich-basel-wo-gibt-es-am-meistenschwarzfahrer). So wird von den Befragten die tatsächliche Entdeckungswahrscheinlichkeit offensichtlich unterschätzt.

18 Wegen des Querschnitts kann nicht geklärt werden, in welche Richtung eine eventuelle Kausalität verläuft. Mit Längsschnittdaten müsste untersucht werden, ob es sich um Selbstselektion in delinquente Gruppen handelt oder ob die Gruppe selbst aufgrund der Kontexte und Gelegenheiten delinquente Normen und Verhaltensweisen entwickelt. Erwähnenswert ist, dass mit der Dichte an delinquenten Kontakten die negative Korrelation mit dem Reputationsverlust abnimmt. Dieser Zusammenhang ist am stärksten für Schwarzfahren $(r=-0,27)$ und am schwächsten für Ladendiebstahl $(r$ $=-0,09)$. 


\begin{tabular}{|c|c|c|c|c|c|c|}
\hline \multirow{2}{*}{ 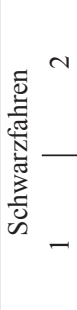 } &  & 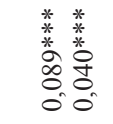 & 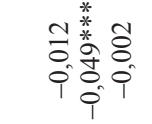 & \multicolumn{2}{|l|}{$\begin{array}{l}\text { * } \\
\text { *ैं } \\
0 \\
0 \\
0\end{array}$} & 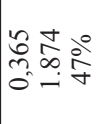 \\
\hline & 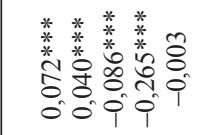 & & & & & 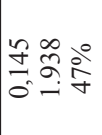 \\
\hline \multirow{2}{*}{ 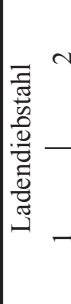 } & 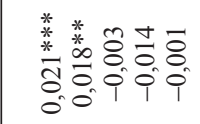 & 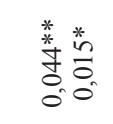 & 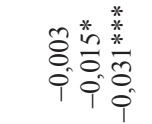 & $\begin{array}{l}* \\
\stackrel{*}{n} \\
0 \\
0\end{array}$ & 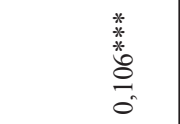 & 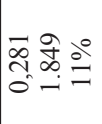 \\
\hline & 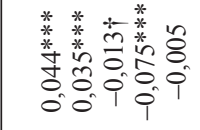 & & & & & m̋ \\
\hline \multirow{2}{*}{ 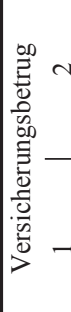 } & 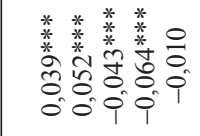 & 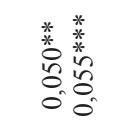 & 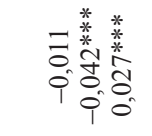 & $\begin{array}{l}* \\
* \\
\tilde{e}_{0}^{*} \\
0 \\
0 \\
0\end{array}$ & 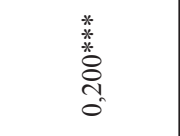 & 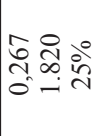 \\
\hline & 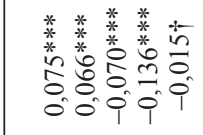 & & & & & 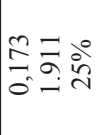 \\
\hline \multirow{2}{*}{ 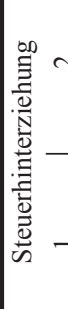 } & 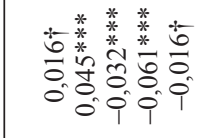 & 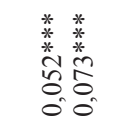 & 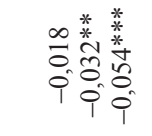 & 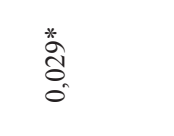 & $\begin{array}{l}* \\
* \\
* \\
\infty \\
m \\
0 \\
0\end{array}$ & 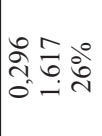 \\
\hline & 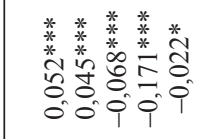 & & & & & 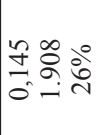 \\
\hline 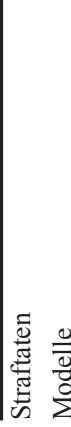 & 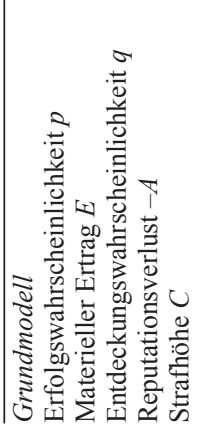 & 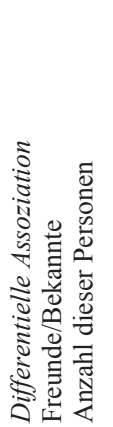 & 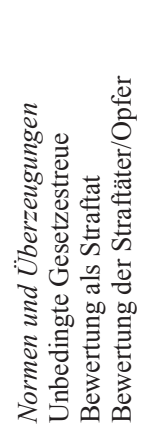 & 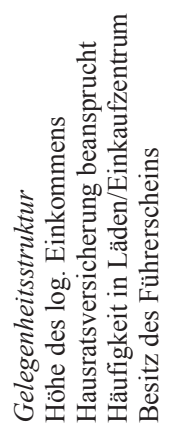 & 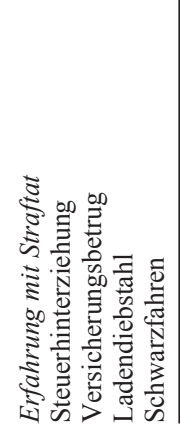 & 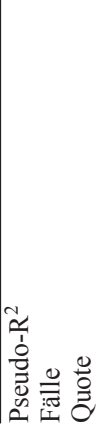 \\
\hline
\end{tabular}


sozialer Anerkennung einhergeht. Auch werden die Straftaten in dem Fall auch nicht als verwerflich erachtet, sondern - im Gegenteil - als ein mit Sicherheit gewinnbringendes Handeln, das ohne negative Konsequenzen bleibt.

Bezüglich der normativen Überzeugungen und Bewertungen weist der Einfluss der kategorischen Gesetzestreue zwar in die theoretisch erwartete Richtung, ist aber in allen Fällen statistisch insignifikant. Wird die jeweilige Straftat als verwerflich erachtet, dann ist die Neigung diese Straftat auszuüben signifikant gering. Die Bewertung der Straftäter bzw. Opfer hat eine jeweils unterschiedliche Auswirkung auf die Delinquenz. Personen, die davon überzeugt sind, dass Steuerhinterzieher der Gesellschaft schaden, sehen eher von einer Steuerhinterziehung ab. Personen, die glauben, Versicherungen seien gierig, neigen eher dazu die eigene Hausratsversicherung zu betrügen als Personen, die gegenteiliger Ansicht sind. Befragte, die angaben, dass es nicht schlimm sei, ein großes Warenhaus zu bestehlen, sind eher bereit Ladendiebstahl zu begehen. Und schließlich fahren Personen eher schwarz, wenn sie glauben, die Preise der öffentlichen Verkehrsmittel in Bern seien zu hoch.

Insgesamt kann an dieser Stelle für die Befragten zunächst festgehalten werden, dass bei Kontrolle normativer Überzeugungen und Bewertungen einerseits und differentieller Assoziation andererseits die grundlegenden Mechanismen der Entscheidung bedeutsam sind, Straftaten sind in der Regel keine alltäglichen Handlungsroutinen, die automatisch-spontan ausgeführt werden. Offensichtlich handelt es sich eher um zweckrational deliberierte Entscheidungen. Auch bei Kontrolle von Gelegenheitsstrukturen und früheren Erfahrungen mit den Straftaten entscheiden und handeln die Befragten typischerweise rein zweckrational. Sind Normen gegen diese Straftaten internalisiert, dann kann angesichts unserer Ergebnisse davon ausgegangen werden, dass unhinterfragt im Sinne der Wertrationalität von Delinquenz abgesehen wird.

Die Gelegenheitsstruktur hat generell einen bedeutsamen Einfluss auf die Intention, eine Straftat zu begehen. Je höher das zu versteuernde Einkommen ist, desto eher wird eine Steuerhinterziehung erwogen. Je häufiger eine Hausratsversicherung in Anspruch genommen wurde, desto geringer ist die Neigung, diese zu betrügen. Wer häufig in Läden der Berner Innenstadt und in Einkaufszentren einkaufen geht, erliegt offenkundig eher der Versuchung Ladendiebstahl zu begehen. Und schließlich sind Besitzer eines Führerscheins eher geneigt schwarzzufahren als Personen ohne Berechtigung ein Kraftfahrzeug zu führen. ${ }^{19}$

Schließlich hat die Erfahrung mit Straftaten einen positiven Einfluss auf die Intention, diese Straftaten auch in Zukunft zu begehen. Wer in der Vergangenheit Steuern hinterzogen, die Hausratsversicherung betrogen, im Warenhaus Güter ohne Bezahlung mitgenommen oder öffentliche Verkehrsmittel ohne gültiges Ticket benutzt hat, ist vergleichsweise eher geneigt diese Straftaten wieder zu begehen. Offensichtlich kann traditionelles Handeln bei Straftaten auftreten, wenn die Handlung eingeübt ist und dann gewohnheitsmäßig auftritt. Diese Schlussfolgerung ist allerdings unter Vorbehalt zu sehen. Denn die eigenen Misserfolge bei diesen Straftaten (oder die Misserfolge anderer Personen im Freundes- und Bekanntenkreis) wiesen allerdings viele fehlende Angaben auf. Was die eigene Erfahrung anbelangt, kann vermutet werden, dass diese aus Scham nicht berichtet wurde, und was die Erfahrung anderer Personen im eigenen Netzwerk angeht, wussten offensichtlich viele Befragte nicht, ob und wie oft andere bei Straftaten erwischt und verurteilt wurden. Daher konnte nicht überprüft werden, ob eigene Erfahrungen oder die Erfahrungen anderer mit der Straf-

19 Warum die frühere Inanspruchnahme der Hausratsversicherung negativ mit der Neigung korreliert, diese zu betrügen, kann den Daten nicht entnommen werden. Dass der Besitz des Führerscheins mit Schwarzfahren einhergeht, lässt sich zum Teil damit erklären, dass diese Besitzer oftmals kein ÖV-Abonnement haben. 
verfolgung einen Einfluss auf die Intention für das Begehen von Straftaten haben. Das müsste in Zukunft mit angemessenen Längsschnittdaten untersucht werden, um sicherzugehen, ob es Gruppen gibt, die aufgrund positiver Erfahrungen routiniert Straftaten begehen.

\section{Zusammenfassung und Schlussfolgerung}

Ziel des vorliegenden Beitrags ist, die Arbeit von Becker / Mehlkop (2006) fortschreibend, eine theoretisch elegante und sparsame Beschreibung und Erklärung der Delinquenz als soziales Handeln - unter Einbezug der Täter, Opfer, Strafverfolger und Netzwerke als Akteure - im sozialen Kontext vorzuschlagen und empirisch zu überprüfen. Kriminelles Handeln wurde als zweckrationales Handeln modelliert, wobei der subjektive Sinn dieses sozialen Handelns nicht in jedem Fall rein zweckrational sein muss, sondern auch wertrational oder traditional und in speziellen Fällen gar affektuell sein kann. In letzteren Fällen dürfte das strafbare Handeln automatisch-spontan, die Kosten-Nutzen-Abwägung implizierend, ohne vorherige Deliberation von Vor- und Nachteilen erfolgen. Diese Vermutung zum subjektiven Sinn hat sich für die ersten drei Typen sozialen Handelns empirisch bestätigt. Jedoch bedarf es weiterer Anwendung für hier nicht beobachtete Straftaten. So könnten politisch motivierte Terrorakte mit Wertrationalität oder Gewaltkriminalität mit affektuellem Handeln aus Hass umschrieben werden Des Weiteren hat es sich als sinnvoll erwiesen, den strukturell-individualistischen Kern der Theorie um kompatible Theorien - wie etwa die Theorie differentieller Assoziation, Erweiterung der Anomietheorie um Gelegenheitsstrukturen, sozial-kognitive Lerntheorie, Theorie kognitiver Rationalität, Theorie begrenzter Rationalität, Theorie sozialer Produktionsfunktion, ,Prospect theory ' und ,Theory of human agency " anzureichern, um eine realistischere mechanismen- und prozessbasierte Erklärung kriminellen Handelns zu erzielen.

Die vorgelegten Analysen haben ihre Grenzen, die vor allem mit der Querschnittbetrachtung verbunden sind und der Aufdeckung endogener Kausalitäten gewisse Grenzen setzen. Aufgrund der für die Befragten sicherlich heiklen Fragen zu den einzelnen Delikten und ihren Korrelaten sind nicht nur sozial erwünschte Antworten möglich, sondern wenn die abhängigen und unabhängigen Variablen zugleich in einer Befragung erhoben werden, sind Konsistenzzwänge und Rationalisierungen bei den Befragten nicht ausgeschlossen. Davon sind direkte Messungen der Mechanismen, die bei Entscheidungen für oder gegen eine bestimmte Straftat eine besondere Rolle spielen, sicherlich besonders betroffen. In weiteren methodischen Untersuchungen müsste diese hier noch offene Frage geklärt werden.

Eine Modellierung kriminellen Handelns im Längsschnitt - d.h. in der Logik des Lebensverlaufs - würde wohl zusätzliche Erkenntnisse liefern und die Erklärung von Kriminalität nach Lebensspannen mit entsprechender Etikettierung wie Jugend- oder Alterskriminalität obsolet machen. Entscheidend sind zeitlich-kontinuierliche Messungen von Gelegenheitsstrukturen und die Erfassung zeitveränderlicher Nutzen und Kosten im Lebensverlauf sowie die Beschreibung des kriminellen Handelns als stochastisches Ereignis. Ob die vorgeschlagene Theorie für andere Straftaten wie etwa Mord oder organisierte Kriminalität angewendet werden kann, ist eine empirisch zu überprüfende Angelegenheit. Möglicherweise muss sie - wie für jeden anderen Typus von Straftaten - expliziert werden. Jedoch kann erwartet werden, dass sich das vorgeschlagene Basismodell als ausbaufähig und erklärungskräftig erweist. Der generalisierbare Nachweis, dass auch bei Straftaten Wertrationalität, Gewohnheiten und Affekt mit automatisch-spontaner Entscheidung und Handlung einhergehen, wird wohl nur dann möglich sein, wenn neben normkonformen und gelegentlich straffällig werdenden Personen auch gewohnheitsmäßige Straftäter und Berufskriminelle befragt werden können (Gambetta 1993). 


\section{Literatur}

Bandura, A. (2001): Social Cognitive Theory, in: Annual Review of Psychology 52, S. 1-26.

Becker, G.S. (1968): Crime and Punishment: An Economic Approach, in: The Journal of Political Economy 76, S. 169-221.

Becker, R. (2006): Selective Response to Questions about delinquency, in: Quality \& Quantity 40, S. 483-498.

Becker, R. / G. Mehlkop (2006): Social Class and Delinquency, in: Rationality \& Society 17, S. 195-235.

Becker, R. / R. Imhof / M. Raimann (2007): Kriminalität als rationale Wahlhandlung, in: Schweizerische Zeitschrift für Soziologie 33, S. 237-260.

Becker, R. / G. Mehlkop (2011): Effects of prepaid monetary incentives on mail survey response rates and on self-reporting about delinquency - Empirical findings, in: Bulletin of Sociological Methodology 111, S. 5-25.

Block, M.K. / R.C. Lind (1975): Crime and Punishment Reconsidered, in: The Journal of Legal Studies 4, S. 241-247.

Boudon, R. (2003): Beyond Rational Choice Theory, in: Annual Review of Sociology 29, S. 1-21.

Brame, R. / R. Paternoster (2003): Missing Data Problems in Criminological Research: Two Case Studies, in: Journal of Quantitative Criminology 19, S. 55-78.

Coleman, J.S. (1990): Foundations of Social Theory, Cambridge / MA.

Cloward, R.A. / L.E. Ohlin (1961): Delinquency and opportunity, London.

Cook, P. J. (1986): The Demand and Supply of Criminal Opportunities. in: M. Tonry / N. Morris (Hrsg.), Crime and Justice: An Annual Review of Research (Vol. 7), Chicago / IL, S. 1-28.

Dahlbäck, O. (2003): Analyzing Rational Crime - Models and Methods, Dordrecht.

Dillman, D.A. (2000): Mail and Internet Surveys: The Tailored Design Method, New York / NY.

Durkheim, É. (1976): Die Regeln der soziologischen Methode, Neuwied / München.

Ehrlich, I. (1973): Participation in Illegitimate Activities: A Theoretical and Empirical Investigation, in: Journal of Political Economy 81, S. 521-565.

Erikson, R. / J.H. Goldthorpe (1992): The Constant Flux, Oxford.

Esser, H. (1999): Soziologie. Spezielle Grundlagen, Band 1: Situationslogik und Handeln. Frankfurt / Main.

Fehr, E. / U. Fischbacher (2004): Third-party punishment and social norms, in: Evolution and Human Behavior 25, S. 63-87.

Gambetta, D. (1993): The Sicilian Mafia. The business of private protection, Cambridge / MA.

Grogger, J. (1991): Certainty vs. Severity of Punishment, in: Economic Inquiry 29, S. 297-310.

Hinz, T. / K. Auspurg (2011): Gruppenvergleiche bei Regressionen mit binären abhängigen Variablen Probleme und Fehleinschätzungen am Beispiel von Bildungschancen im Kohortenverlauf, in: Zeitschrift für Soziologie 40, S. 62-73.

Huizinga, D. / D.S. Elliott (1986): Reassessing the Reliability and Validity of Self-Report Delinquency Measures, in: Journal of Quantitative Criminology 2, S. 293-327.

Kahneman, D. (2011): Schnelles Denken, langsames Denken, München.

Köllisch, T. / D. Oberwittler (2004): Wie ehrlich berichten männliche Jugendliche über ihr delinquentes Verhalten? Ergebnisse einer externen Validierung, in: Kölner Zeitschrift für Soziologie und Sozialpsychologie 56, S. 709-736.

Kroneberg, C. / I. Heintze / G. Mehlkop (2010): The Interplay of Moral Norms and Instrumental Incentives in Crime Causation, in: Criminology 48, S. 259-294. 
Lauritsen, J.L. (1998): The Age-Crime-Debate: Assessing the Limits of Longitudinal Self-Report Data, in: Social Forces 7, S. 127-154.

Lindenberg, S. (1989): Social Production Functions, Deficits, and Social Revolutions, in: Rationality and Society 1, S. 51-77.

Lochner, L. (2004): Education, Work, And Crime: A Human Capital Approach, in: International Economic Review 45, S. 811-843.

Loughran, T.A. / H. Nguyen / A.R. Piquero / J. Fagan (2013): The Returns to Criminal Capital, in: American Sociological Review 78, S. 925-948.

Ludwig-Mayerhofer, W. (2005): Arbeitslosigkeit, in: M. Abraham / T. Hinz (Hrsg.), Arbeitsmarktsoziologie, Wiesbaden, S. 199-239.

Manski, C. (2004): Measuring Expectations, in: Econometrica 72, S. 1329-1376.

Matsueda, R.L. / D.A. Kreager / D. Huizinga (2006): Deterring Delinquents: A Rational Choice Model of Theft and Violence, in: American Sociological Review 71, S. 95-122.

Matsueda, R.L. (2013): Rational Choice Research in Criminology: A Multi-Level Framework, in: R. Wittek / T.A.B. Snijeders / V. Nee (Hrsg.), The Handbook of Rational Choice Social Research, Stanford /CA, S. 283-321.

Matsueda, R.L. / R. Gartner / I. Piliavin / M. Polakowski (1992): The Prestige of Criminal and Conventional Occupations: A Subcultural Model of Criminal Activity, in: American Sociological Review 57, S. $752-770$.

McCarthy, B. / J. Hagan (2001): When Crime pays: Capital, Competence and Criminal Success, in: Social Forces 79, S. 1035-1059.

Mehlkop, G. (2011): Kriminelles Verhalten als rationale Handlungswahl, Wiesbaden.

Mehlkop, G. / R. Becker (2007): Zur Wirkung monetärer Anreize auf die Rücklaufquote in postalischen Befragungen zu kriminellen Handlungen. Theoretische Überlegungen und empirische Befunde eines Methodenexperiments, in: Methoden - Daten - Analysen 1, S. 5-24.

Mehlkop, G. / Becker, R. (2004): Soziale Schichtung und Delinquenz. Eine empirische Anwendung eines Rational Choice-Ansatzes mit Hilfe von Querschnittsdaten des ALLBUS 1990 und 2000, in: Kölner Zeitschrift für Soziologie und Sozialpsychologie 56. S. 95-126.

Merton, R.K. (1949): Social Theory and Social Structure, Glencoe.

Nagin, D.S. / G. Pogarsky (2001): Integrating Celerity, Impulsivity, And Extralegal Sanction Threats Into A Model of General Deterrence: Theory And Evidence, in: Criminology 39, S. 865-891.

Niggli, M.A. (1994): Rational Choice Theory and Crime Prevention, in: Studies on Crime and Crime Prevention 3, S. 83-103.

Ormel, J. / S. Lindenberg / N. Steverink / L.M. Verbrugge (1999): Subjective well-being and social production functions, in: Social Indicator Research 46, S. 61-90.

Paternoster, R. / G. Pogarsky (2009): Rational Choice, Agency and Thoughtfully Reflective Decision Making: The Short and Long-Term Consequences of Making Good Choices, in: Journal of Quantative Crimonology 25, S. 103-127.

Paternoster, R. / L.E. Saltzman / G.P. Waldo / T.G. Chiricos (1983): Perceived Risk and Social Control: Do Sanctions Really Deter?, in: Law \& Society Review 17, S. 457-479.

Piliavin, I. / C. Thornton / R. Gartner / R.L. Matsueda (1986): Crime, Deterrence, and Rational Choice, in: American Sociological Review 51, S. 101-119.

Piquero, A.R. / R. Macintosh / M. Hickman (2002): The Validity of a Self-Reported Delinquency Scale. Comparisons Across Gender, Age, Race, and Place of Residence, in: Sociological Methods and Research 30, S. 492-529.

Piquero, A.R. / T. Brezina / M.G. Turner (2005): Testing Moffitt's Account of Delinquency Abstention, in: Journal of Research in Crime and Delinquency 42, S. 27-54. 
Pogarsky, G. / A.R. Piquero / R. Paternoster (2004): Modeling Change in Perceptions about Sanctions Threats: The Neglected Linkage in Deterrence Theory, in: Journal of Quantitative Criminology 20, S. 343-369.

Sampson, R.J. / J.H. Laub (1992): Crime and Deviance in the Life Course, in: Annual Review of Sociology 18, S. 63-84.

Sampson, R.J. / J.H. Laub (2003): Desistance from Crime over the Life Course, in: J.T. Mortimer / M.J. Shanahan (Hrsg.), Handbook of the Life Course, New York / NY, S. 295-309.

Schulz, S. (2013): Individual Differences in the Deterrence Process: Which Individuals Learn (Most) from Their Offending Experiences?, in: Journal of Quantitative Criminology 30, S. 215-236.

Stocké, V. (2010): Der Beitrag der Theorie rationaler Entscheidung zur Erklärung von Bildungsungleichheit und Bildungsarmut, in: G. Quenzel / K. Hurrelmann (Hrsg.), Bildungsverlierer. Neue Ungleichheiten, Wiesbaden, S. 73-95.

Sutherland, E.H. (1968): Die Theorie der differentiellen Kontakte, in: F. Sack / R. König (Hrsg.), Kriminalsoziologie, Frankfurt / Main, S. 395-399.

Sykes, G. / D. Matza (1957): Techniques of Neutralization: A Theory of Delinquency, in: American Sociological Review 22, S. 664-670.

Thornberry, T.P. / M.D. Krohn (2000): The Self-Report Method for Measuring Delinquency and Crime, in: Measurement and Analysis of Crime and Justice 4, S. 33-83.

Urban, D. (1993): Logit-Analyse, Stuttgart.

Ward, D.A. / M.C. Stafford / L.N. Gray (2006): Rational Choice, Deterrence, and Theoretical Integration, in: Journal of Applied Psychology 36, S. 571-585.

Weber, M. (1922): Wirtschaft und Gesellschaft, Tübingen.

Weisburd, D. / A.R. Piquero (2008): How Well Do Criminologists Explain Crime? Statistical Modeling in Published Studies, in: Crime and Justice 37, S. 453-502.

Wikström, P.-O.H. (2006): Individuals, settings, and acts of crime: situational mechanisms and the explanation of crime, in: P.-O.H. Wikström / R.J. Sampson (Hrsg.), The Explanation of Crime. Context, Mechanisms, and Development, New York / NY, S. 61-107.

Zhang, L. / S.F. Messner (2000): The Effects of Alternative Measures of Delinquent Peers on Self-Reported Delinquency, in: Journal of Research in Crime and Delinquency 37, S. 324-337.

Prof. Dr. Rolf Becker Universität Bern Institut für Erziehungswissenschaft Fabrikstrasse 8 CH-3012 Bern rolf.becker@edu.unibe.ch 